\title{
Venus Mesosphere and Thermosphere
}

\author{
II. Global Circulation, Temperature, and Density Variations
}

\author{
S. W. BOUGHER,,$\uparrow$ R. E. DICKINSON,$\ddagger$ E. C. RIDLEY,§ R. G. ROBLE, ${ }^{*}$ \\ A. F. NAGY,"AND T. E. CRAVENS" \\ ${ }^{*}$ High Altitude Observatory, $\dagger$ Advanced Study Program, $\ddagger$ Atmospheric Analysis and Prediction, and \\ $\S$ Scientific Computing Division, National Center for Atmospheric Research, ${ }^{1}$ P.O. Box 3000, Boulder, \\ Colorado 80307; and "Space Physics Research Laboratory, 2455 Hayward, University of Michigan, \\ Ann Arbor, Michigan 48109
}

Received February 13, 1986; revised July 11, 1986

\begin{abstract}
Recent Pioneer Venus observations have prompted a return to comprehensive hydrodynamical modeling of the thermosphere of Venus. Our approach has been to reexamine the circulation and structure of the thermosphere using the framework of the R. E. Dickinson and E. C. Ridley (1977, Icarus 30, 163-178), symmetric two-dimensional model. Sensitivity tests were conducted to see how large-scale winds, eddy diffusion and conduction, and strong $15-\mu \mathrm{m}$ cooling affect day-night contrasts of densities and temperatures. The calculated densities and temperatures are compared to symmetric empirical model fields constructed from the Pioneer Venus data base. We find that the observed day-to-night variation of composition and temperatures can be derived largely by a wavedrag parameterization that gives a circulation system weaker than predicted prior to Pioneer Venus. The calculated mesospheric winds are consistent with Earth-based observations near 115 $\mathrm{km}$. Our studies also suggest that eddy diffusion is only a minor contributor to the maintenance of observed day and nightside densities, and that eddy coefficients are smaller than values used by previous one-dimensional composition models. The mixing that occurs in the Venus thermosphere results from small-scale and large-scale motions. Strong $\mathrm{CO}_{2} 15-\mu \mathrm{m}$ cooling buffers solar perturbations such that the response by the general circulation to solar cycle variation is relatively weak. 1986 Academic Press, lnc.
\end{abstract}

\section{INTRODUCTION}

New information obtained from Pioneer Venus (PV) spacecraft observations makes it appropriate to return to comprehensive hydrodynamical model studies of the thermospheric circulation of Venus. The general features of the inferred mean subsolarto-antisolar circulation were previously predicted in a series of numerical studies by Dickinson and Ridley (1972, 1975, 1977). However, these calculations failed to reproduce the observed day-night temperature contrast, specifically the very cold nightside temperatures. The Dickinson and Ridley (1977) model (or DRM) also pre-

\footnotetext{
1 The National Center for Atmospheric Research is sponsored by the National Science Foundation.
}

dicted strong $400 \mathrm{~m} \mathrm{sec}^{-1}$ winds which caused a considerable dayside depletion and nightside enhancement of $\mathrm{O}$ and $\mathrm{CO}$ densities. It has become apparent from the PV data that the actual structure in Venus's upper atmosphere has weaker day-night contrasts of these constituents and is more complex than can be modeled by a purely symmetric subsolar-to-antisolar circulation (Mayr et al., 1980, 1985). A summary of the differences between DRM model calculations and Pioneer Venus observations was provided in Fig. 1 of Dickinson and Bougher (1986), hereafter referred to as Part I.

The discrepancies between hydrodynamic model predictions and PV observations have been puzzling. The large-scale circulation and its effects on temperature 
and composition fields are included in the self-consistent, coupled primitive equation model of DRM. However, small-scale mixing processes inferred from observed atmospheric wave signatures (Seiff et al., 1980; Seiff, 1982) were not incorporated in any systematic manner. On the other hand, one-dimensional composition models of von Zahn et al. (1980), Stewart et al. (1980), and Massie et al. (1983) have been used to examine the influence of eddy mixing on densities; these neglect any self-consistent incorporation of large-scale dynamical effects. Simpler hydrodynamic models (e.g, Mayr et al., 1980, 1985) are highly parameterized and, like one-dimensional models, cannot examine the strong feedback among temperature, composition, and wind fields. All of these previous model descriptions incorporate important processes of the actual Venus thermosphere. However, a new selfconsistent and quantitative simulation of the Venusian upper atmosphere is ultimately needed that combines the key features of these separate models.

As discussed in Part $I$, there are several questions to consider in the development of an improved hydrodynamical model. First, how can we obtain a global average temperature structure with exospheric values near $210^{\circ} \mathrm{K}$ as observed? Second, how can we model the day-night temperature contrast, which is nearly $200^{\circ} \mathrm{K}$ at exospheric heights but rather small at $100 \mathrm{~km}$ and below? Third, how can we obtain atomic oxygen concentrations as large and with as little diurnal variation along constant pressure surfaces about the equator as those observed? It is also important to be able to reproduce the asymmetrical signatures observed for the light species, particularly $\mathrm{He}$ and $\mathrm{H}$ densities.

The calculation of a reasonable global average temperature structure was addressed in Part $I$ as a first step in the simulation of the dynamics and observed characteristics of the Venus thermosphere. It was found that cooling by eddy mixing alone is not sufficient to balance EUV heating with effi- ciencies greater than $10 \%$ without the use of excessively large eddy coefficients, even assuming the maximum possible eddy cooling. This cooling is even less effective if we assume a partial compensation of frictional heating and cooling. However, an improved thermal balance can be obtained with enhanced $15-\mu \mathrm{m}$ cooling. For our final reference calculation, we adopted a large but not completely improbable rate coefficient for enhancing $\mathrm{CO}_{2}$ radiation through collisions with ambient atomic oxygen, $K_{\mathrm{CO}_{2}-\mathrm{O}}=8 \times 10^{-13} \mathrm{~cm}^{3} \mathrm{sec}^{-1}$ at $300^{\circ} \mathrm{K}$, in conjunction with a $9.5 \%$ EUV efficiency to obtain the observed temperatures. This value is roughly double that stated in Part $I$, as required to allow for a minor coding error found in the one-dimensional radiative transfer model above $140 \mathrm{~km}$. The bottom four entries of Table $I$ and the ordinate in Fig. 4 of Part I should be similarly adjusted. With these adjustments, all other conclusions and diagnostics are largely unchanged; in particular, heating efficiencies much in excess of 10-12\% are presently inconsistent with any known cooling mechanism for Venus's atmosphere. This corrected final reference calculation provides the basis for deriving the infrared heating and $15-\mu \mathrm{m}$ cooling inputs required for the improved hydrodynamical model simulation (see Sect. 2).

Having established the parameters most suitable for reproducing the observed global mean temperatures, we investigate here the day-night temperature contrasts and compositional distributions. Several plausible alterations of the DRM large-scale circulation have been suggested to improve its agreement with the observed Venus thermospheric structure. The mechanism explored here is a global circulation system weaker than predicted by DRM (von Zahn et al., 1983). Comparison of Dickinson and Ridley (1975) and the later DRM model output fields shows an increase in dayside atomic $\mathrm{O}$ densities and a corresponding decrease on the nightside. These changes are presumably caused by a weakening of the 
day-to-night winds in response to a reduced dayside heating in this sequence of model calculations. Thus, a sufficiently weakened wind structure can maintain observed $\mathrm{O}$ and $\mathrm{CO}$ densities on the dayside while reducing the buildup on the nightside. Furthermore, the DRM has established that the nightside heating comes primarily from the circulation, mostly from adiabatic compression due to descending nightside winds, but also from thermal advection across the terminator. Hence, slower winds would also result in cooler nightside temperatures. A suitable mechanism for slowing the winds would change the distribution of atmospheric constituents and temperatures about the planet. Terrestrial mesopause wave-breaking models serve as a guide for possible momentum drag schemes (Lindzen, 1981; Holton, 1982). Eddy viscosity and Rayleigh friction may approximate the effects of wave drag on the mean flow.

Mayr et al. (1985) have proposed a different scheme to match the observed daynight distribution of composition and temperatures. They constructed a spectral model with the diurnal and higher order components of the thermospheric structure, and found that a particular combination of strong vertical eddy diffusion and retrograde zonal winds might modify the mean subsolar-to-antisolar circulation, giving strong winds $\left(300 \mathrm{~m} \mathrm{sec}^{-1}\right)$ toward the terminator. Their model requires large vertical eddy diffusion $\left(K=6 \times 10^{3} \mathrm{~m}^{2} \mathrm{sec}^{-1}\right)$ at altitudes above $130 \mathrm{~km}$ to counteract the effects of the large-scale winds. With such diffusion, they find a reasonable agreement between observed and modeled phases and magnitudes of global temperatures and densities. Below $130 \mathrm{~km}$, eddy viscosity is weakened to permit a redistribution of heat and atmospheric constituents about the planet.

Another possibility, suggested by DRM and von Zahn et al. (1983), is that a strong rotational wind may provide a "return flow" below $100 \mathrm{~km}$ to transport $\mathrm{O}$ and $\mathrm{CO}$ (and other light species) from the nightside back to the dayside without significantly affecting the global temperatures. Such a scheme could jointly improve the density distributions while retaining the strong (300-400 $\mathrm{m} \mathrm{sec}-1$ ) terminator neutral winds.

The DRM code has been completely redeveloped in recent years to run on the NCAR CRAY computers. Apparently, previously neglected physical processes, such as those described above, are required to explain the observed day-night thermal and density contrasts observed in Venus's upper atmosphere. We make use of the large-scale circulation scheme of the previous DRM model as a framework upon which such model processes as eddy diffusion, eddy or viscous wave drag, and 15$\mu \mathrm{m}$ radiational cooling are systematically examined. The two-dimensional model framework that we adopt here is only capable of examining the symmetric component of Venus's thermospheric circulation and structure.

Our improved model gives a weakened global circulation whose corresponding densities and temperatures match empirical model fields constructed from PV data ( $\mathrm{He}$ din et al., 1983; Keating et al., 1984). We assume that large-scale motions and smallscale mixing processes both modify densities and temperatures, as they apparently do in the Earth's thermosphere (Mayr et al., 1978). Various model parameterizations and new data are incorporated into our present hydrodynamical calculations. In particular, an improved perturbation 15$\mu \mathrm{m}$ cooling scheme is used to provide accurate cooling over the globe, based upon the corrected final reference model results of Part I. Sensitivity tests show the relative roles of large-scale winds and eddy diffusion in determining observed densities. We attempt to improve both the calculated day-night temperature and the density contrasts.

We find that the composition and temperature variations observed by Pioneer Venus can be approximated using a wave-drag 
parameterization that gives a circulation system weaker than predicted by DRM. The calculated mesospheric winds near 115 $\mathrm{km}$ are consistent with the Earth-based observations of Betz (1982). Our hydrodynamic model calculations suggest that eddy coefficients are smaller than values used by previous one-dimensional composition models, and that eddy diffusion is only a minor contributor to the maintenance of observed nightside densities.

\section{CURRENT FORMULATION OF THE TWO-DIMENSIONAL MODEL}

\subsection{Two-Dimensional Model Equations aNd MECHaNiCS}

It is often argued that the predictions of detailed hydrodynamical models might be represented by one-dimensional eddy mixing models. Several one-dimensional composition models have been developed using eddy mixing to simulate vertical motions (von Zahn et al., 1980; Stewart et al., 1980; Massie et al., 1983; Shimazaki et al., 1984). Dickinson and Ridley (1977) indicate that this approach might be reasonable provided that composition alone is considered. However, the motions parameterized in these eddy mixing models control not only densities, but also drastically alter the thermal structure. A significant feedback is present among the temperature, composition, and wind fields, such that the independent calculation of any field is of questionable significance. The use of a coupled chemicaldynamical model like DRM is thus necessary for a more realistic simulation of Venus's thermospheric fields.

The two-dimensional DRM model was designed to calculate temperature, composition, and winds of the upper mesosphere and thermosphere of Venus using a nonlinear time-dependent formulation. Chemistry and dynamics are coupled through transport equations. Such a model of the largescale circulation circumvents the primary limitation of the one-dimensional calculations, which do not use motions derived from first principles. The model winds modify the horizontal variation of temperatures and densities, thereby enabling a coupled set of global fields to be calculated.

Our improved model assumes (as in DRM) that all variables depend only on altitude (95-210 km) and on distance from the subsolar point. This geometry, symmetric about the Sun-Venus axis, effectively models conditions near the equator where most of the Pioneer Venus in situ data were obtained. The vertical coordinate for calculation is defined to be log-pressure, $z=$ $\ln \left(p_{0} / p\right)$, measured from the $p_{0}$ reference level of $5 \times 10^{-3} \mu \mathrm{bar}$, and spanning the range $z=-10$ to 7 . The horizontal coordinate is colatitude $\theta$ (solar zenith angle) with a $5^{\circ}$ grid ranging from local noon to midnight. There are 137 vertical levels in increments of $\Delta z=0.125$. Because of the slow planetary rotation, Coriolis effects are neglected.

The hydrodynamical system consists of four prognostic equations, respectively, for horizontal motion $u$, temperature $T$, and the distributions of $\mathrm{O}$ and $\mathrm{CO}$, with two additional diagnostic equations representing continuity and hydrostatic balance. $\mathrm{CO}_{2}$ is calculated passively as the remainder of the total density. Winds are driven by absorption of EUV, UV, and IR radiation, which provides molecular heating. The EUV and UV radiation also dissociates $\mathrm{CO}_{2}$ into $\mathrm{O}$ and $\mathrm{CO}$ products. The near-infrared heating and reference $15-\mu \mathrm{m}$ cooling fields are supplied by the detailed one-dimensional radiative transfer model calculations described in Part 1.

Additional terms are incorporated into some equations to represent the new, physically motivated parameterizations introduced briefly in Section I. The equation of horizontal motion is essentially that given by Dickinson and Ridley (1975), modified now by a wave-drag term proportional to $u$ :

$$
\lambda=\lambda_{0} u(p) \text {. }
$$

Details for the prescription of a drag rate $\lambda$ as a function of pressure $p$ are given in Sec- 
tion 3.2. Molecular viscosity $\mu$ is also altered to include an eddy viscosity component, consistent with the prescribed eddy coefficient, which reflects the influence of breaking gravity waves or turbulence on the mean flow. Similarly, the equation determining $\mathrm{O}$ and $\mathrm{CO}$ concentrations is largely unchanged from Dickinson and Ridley (1975), except for an eddy diffusion term which is added for testing. Its log-pressure form is similar to that of Dickinson et al. (1984):

$$
F_{\text {eddy }}=e^{z} \frac{d}{d z}\left(\frac{K(z)}{H^{2}} e^{-z} \frac{d \Psi}{d z}\right) .
$$

Here, $H=$ mean scale height, $\Psi=$ mass mixing ratio for $\mathrm{O}$ or $\mathrm{CO}$, and $K(z)=\mathrm{a}$ specified vertical eddy coefficient profile (in $\left.\mathrm{m}^{2} \mathrm{sec}^{-1}\right)$. Temperature is calculated by the Dickinson and Ridley (1975) thermodynamic equation, now incorporating an improved "two-reference temperature" 15$\mu \mathrm{m}$ cooling parameterization (see Sect. 2.2). Consistent eddy conduction is added to the thermodynamic equation in agreement with the specified vertical eddy coefficient:

$F_{\text {cond }}=\frac{g e^{z}}{P_{0} C_{\mathrm{p}}} \frac{d}{d z}\left(K(z) C_{\mathrm{p}} \rho\left(\frac{g}{C_{\mathrm{p}}}+\frac{1}{H} \frac{d T}{d z}\right)\right)$

where $g=$ gravity, $C_{\mathrm{p}}=$ specific heat, and $\rho$ $=$ mass density. This conduction is found to be relatively minor in controlling temperatures, in accord with the implications of Part $I$ and Section 3.2 in this paper.

The calculations are run to a steady state that is determined by a predefined convergence criteria for each of the four prognostic fields (see Dickinson and Ridley, 1975). For fixed winds, the composition is generally expected to reach a steady state in approximately one Earth day. However, the distribution of composition near the lower boundary and the temperatures and densities deep on the nightside take several days to adjust to equilibrium values. A $300-\mathrm{sec}$ time step is maintained to avoid rapidly growing linear instability.

\subsection{IMPROVEMENTS FOR AN INITIAL Reference Model Calculation}

\section{(A) Fluxes and Cross Sections}

In the course of developing the two-dimensional code for sensitivity studies, we found that a new initial reference model calculation was required to study the effects of updated fluxes and cross sections. Solar maximum fluxes and cross sections were adopted identical to those of the one-dimensional radiative transfer model calculations of Part I, based on early Pioneer Venus conditions, corresponding to "average solar maximum" output for which the $F 10.7-\mathrm{cm}$ flux is $206 \times 10^{-22} \mathrm{~W} \mathrm{~m}^{-2} \mathrm{~Hz}^{-1}$. Besides the calculated $\mathrm{O}, \mathrm{CO}$, and $\mathrm{CO}_{2}$, the fractional $\mathrm{N}_{2}$ density distribution is now specified according to the Hedin et al. (1983) empirical model, giving, a more complete description of the major species in the two-dimensional model.

\section{(B) Infrared Cooling Parameterization}

The one-dimensional model studies of Part I decomposed the global mean calculation into separate dayside and nightside models, with solar heating divided between day and night to simulate hydrodynamical effects. This separation was necessary to allow for the large nonlinearity of the 15$\mu \mathrm{m}$ radiational cooling. Once the observed day-night contrast in Venusian temperatures was reproduced, a consistent global mean temperature structure was obtained by averaging dayside and nightside calculations. The thermal infrared cooling can now be approximated in the two-dimensional model by these same dayside and nightside reference cooling profiles plus a NLTE cool-to-space formulation for deviations from these references. A nonlinear temperature-dependent parameterization is used to interactively calculate total $15-\mu \mathrm{m}$ cooling at other temperatures based upon these externally supplied reference cooling profiles. Such a two-reference temperature scheme provides a more accurate means to 
calculate $15-\mu \mathrm{m}$ cooling over all solar zenith angles.

Appendix A outlines the formulation necessary to incorporate this new $15-\mu \mathrm{m}$ cooling scheme in the two-dimensional model. The explicit ratio of $\mathrm{K}_{\mathrm{CO}_{2}-\mathrm{O}}$ and $\mathrm{K}_{\mathrm{CO}_{2}-\mathrm{CO}_{2}}$ is now calculated, with the $\mathrm{K}_{\mathrm{CO}_{2}-\mathrm{CO}_{2}}$ temperature dependence taken from Simpson et al.

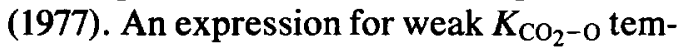
perature dependence, similar to that of Sharma and Nadile (1981), is also given.

\section{(C) Improvements in Chemical}

\section{Prescription}

We reexamined all chemical and $d y$ namical assumptions of DRM for the development of an improved hydrodynamical model simulation. Since we were concerned that the parametrically prescribed neutral chemistry of DRM might be inferior to the more realistic and complete formulation used by Massie et al. (1983), we attempted to match the production and loss $P-L$ terms at $60^{\circ}$ solar zenith angle, given by Massie et al. (1983), with those calculated by the two-dimensional model.

Several minor changes in the two-dimensional model were made to reduce discrepancies in the $P-L$ terms between our calculation and that of Massie et al. (1983) to within $20 \% . \mathrm{N}_{2}, \mathrm{O}$, and $\mathrm{CO}$ photoionization rates are now calculated, providing additional sources of $\mathrm{CO}_{2}^{+}$by ion-molecule and ion-atom interchange. These contributions provide a modest increase in $\mathrm{O}$ production above $160 \mathrm{~km}$, since each ionized species produced eventually recombines to produce one $\mathrm{O}$ and $\mathrm{CO}$ pair. This additional $\mathrm{O}$ and $\mathrm{CO}$ source is augmented by another $40 \%$ (as in Massie et al., 1983), to represent the effects of secondary ionization. Similarly, the multiple dissociation possible from directly ionized $\mathrm{CO}_{2}^{+}$is parameterized in a fashion similar to DRM. Now, however, the threshold wavelength for $100 \%$ yield is raised to $\lambda=600 \AA$, as suggested by Torr et al. (1979). Finally, recombination of atomic oxygen through $\mathrm{O}+\mathrm{O}+\mathrm{CO}_{2}$ is included in our calculations with a notice- able effect upon nightside low-altitude net $O$ production and heating. Roughly $75 \%$ of the resultant excess energy is permitted to be thermalized, with the remainder lost via $\mathrm{O}_{2}\left({ }^{1} \Delta\right)$ air glow.

\section{(D) EUV Heating Balanced by Enhanced 15- $\mu \mathrm{m}$ Cooling}

We use $15-\mu \mathrm{m}$ cooling and EUV efficiency parameters similar to those of the corrected final reference model of Part I to provide net heating in our two-dimensional model. An EUV efficiency of $10 \%$ is used and $\mathrm{K}_{\mathrm{CO}_{2}-\mathrm{O}}=9 \times 10^{-13} \mathrm{~cm}^{3} \mathrm{sec}^{-1}$ at $300^{\circ} \mathrm{K}$ to give improved global mean temperatures that closely match Pioneer Venus observations. This adopted rate coefficient (see Appendix A) is weakly temperature dependent and is about double that estimated from rocket measurements by Sharma and Nadile (1981). An initial reference model calculation independent of any eddy processes is now established.

\section{TWO-DIMENSIONAL MODEL SENSITIVITY STUDIES}

\subsection{Initial Reference Model Results}

Calculations leading to the development of an initial reference model are presented in this section. This framework is then used to conduct sensitivity tests involving eddy processes (wave drag and diffusion) which are capable of changing the observed thermospheric day-night contrasts in our calculations. For case 1, new inputs are applied to the former DRM code, including the previous lowest limit EUV efficiency of 2-22\% and a moderate $\mathrm{O}-\mathrm{CO}_{2}$ enhancement of $15-\mu \mathrm{m}$ cooling $\left(\mathrm{K}_{\mathrm{CO}_{2}-\mathrm{O}}=2 \times 10^{-13}\right.$ $\left.\mathrm{cm}^{3} \mathrm{sec}^{-1}\right)$. Case 1 also incorporates the effects of updated fluxes, current cross sections, and empirical $\mathrm{N}_{2}$ on the thermal structure and resulting thermospheric circulation. For case 2, global energy balance parameters similar to the corrected final reference model of Part I are specified, including a strong enhancement of $15-\mu \mathrm{m}$ cooling and $\varepsilon=10 \%$ heating. The fluxes 


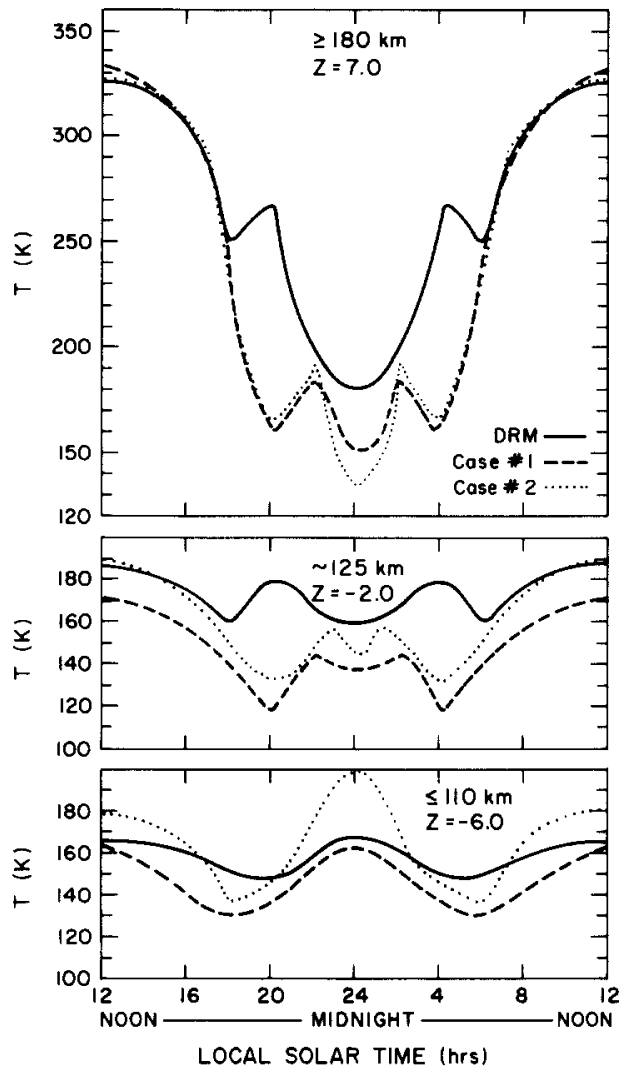

FIG. 1. Initial reference model temperatures at selected pressure levels over all solar zenith angles. Previous DRM temperatures are presented for comparison. Upper case $Z$ (figures) and lower case $z$ (text) both refer to the same log-pressure vertical coordinate.

and cross-sections of case 1 are also used for case 2 . The improved nonlinear $15-\mu \mathrm{m}$ cooling scheme described earlier is also incorporated, along with relatively minor changes in the parameterized chemistry. Case 2 primarily addresses the effects of these improved radiative sources and sinks.

Figure 1 illustrates calculated temperatures on selected pressure level surfaces for the above two cases. Noon and midnight fractional $O$ profiles appropriate for both cases are plotted in Fig. 2, represented by the no drag curves. All comparisons are made with respect to the previous Dickinson and Ridley (1977) calculated model fields. Case 1, with solar maximum fluxes, gives exospheric temperatures slightly warmer at noon than before, as seen in Fig. 1. Depleted davside relative $O$ densities cause the $15-\mu \mathrm{m}$ cooling to be ineffective in cooling dayside temperatures. Nightside temperatures near $125 \mathrm{~km}$ are approximately $20-60^{\circ} \mathrm{K}$ cooler in case 1 than before, with much cooler nightside exospheric temperatures. $\mathrm{CO}_{2}$ cooling is relatively important beyond $\theta=150$ despite the moderate rate coefficient used since calculated $O$ mixing ratios are very large (see Fig. 2). Terminator winds (not shown) are increased to a maximum value of $u=420 \mathrm{~m}$ $\sec ^{-1}$, resulting in this strong enhancement of nightside $\mathrm{O}$ densities (see no drag curves in Fig. 2). Deceleration of horizontal winds and the corresponding nightside "tongue of heating" is shifted toward midnight $(\theta=$ $\left.150^{\circ}\right)$ compared to DRM $\left(\theta=110^{\circ}\right)$, due to the increased dayside heating and stronger day-night winds. Cooler nightside temperatures result from 90 to $130^{\circ}$ solar zenith angles. Evidently, replacing previously used values with solar maximum fluxes and current cross sections makes obtaining a realistic day-night distribution of composition and temperatures even more difficult.

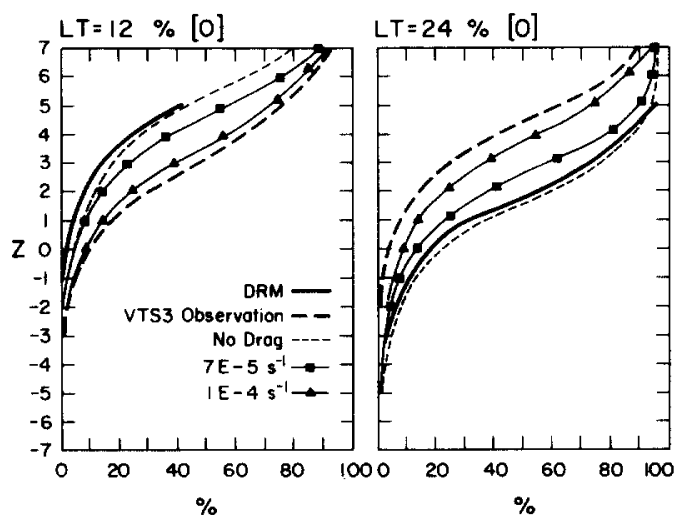

FIG. 2. Venus two-dimensional model atomic oxygen response to viscous drag tests. Drag rates ranging from 0 to $1 \times 10^{-4} \mathrm{sec}^{-1}$ give reduced winds which alter the distribution of atomic $O$ about the planet. Results are compared with previous DRM calculations and Hedin et al. (1983) [VTS3] empirical model values at noon and midnight. 


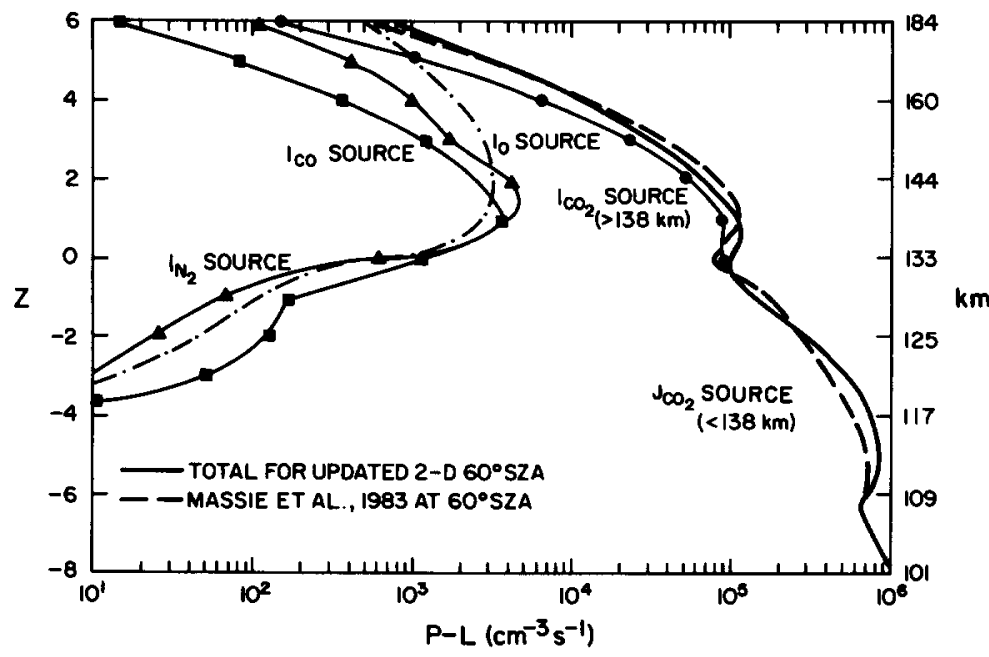

FIG. 3. Production minus loss for $\mathrm{O}$ and $\mathrm{CO}$ at $60^{\circ}$ solar zenith angle. Improved component terms for net production are compared with similar terms included in a one-dimensional composition model. Our two-dimensional model chemistry is now shown to be in excellent agreement with that of Massie et al. (1983) for altitudes above $105 \mathrm{~km}$.

A more realistic global mean thermal structure derived using plausible EUV, IR heating, and $15-\mu \mathrm{m}$ cooling fields is needed. Case 2 examines such a situation using the corrected final reference model parameters of Part I, appropriate for the derivation of observed global mean temperatures. The improved $15-\mu \mathrm{m}$ cooling gives rise to cooler nightside exospheric temperatures, approaching $130^{\circ} \mathrm{K}$. This $20^{\circ} \mathrm{K}$ decrease of temperatures from case 1 is due primarily to the enhanced $\mathrm{O}-\mathrm{CO}_{2}$ rate coefficient used. Warmer dayside temperatures near $125 \mathrm{~km}$ also result from the IR heating. The enhanced day-night temperature gradient drives even stronger terminator winds (up to $440 \mathrm{~m} \mathrm{sec}^{-1}$ ) which deplete dayside $\mathrm{O}$ and $\mathrm{CO}$ further from the observed dayside values, giving fractional $O$ values much like those in case 1 above. This increased buildup of nightside densities provides a more pronounced backpressure that is partially responsible for the further isolation of the deep nightside, and hence cooler temperatures for both cases. Subsidence, providing increased nightside compressional heating, enhances nightside temperatures at $110 \mathrm{~km}$. Any improvements in vertical and horizon- tal temperature gradients are offset by a stronger circulation system that drives calculated densities further from the empirical model values given by Hedin et al. (1983) and Keating et al. (1984).

A modest improvement is made over DRM in calculating dayside neutral densities without greatly affecting the case 2 circulation and temperatures. Figure 3 shows that the newly prescribed ionization sources from $\mathrm{O}, \mathrm{CO}$, and $\mathrm{N}_{2}$ (used in case 2) increase the total net $O$ production above $160 \mathrm{~km}$ by a factor of 2 to 4 , compared to previous DRM calculations. The previously included direct $\mathrm{CO}_{2}^{+}$ionization source is the dominant ionization term at all but these highest altitudes. Below 138 $\mathrm{km}$, ionization gives way to direct $\mathrm{CO}_{2}$ dissociation as the principal source of $\mathrm{O}$ and $\mathrm{CO}$, a process largely undisturbed by our model improvements. Near $110 \mathrm{~km}, \mathrm{O}+$ $\mathrm{O}+\mathrm{CO}_{2}$ recombination provides a significant loss for nightside $O$. Finally, below $105 \mathrm{~km}$ our chemical prescription is inadequate, and minor species chemistry, as suggested by Yung and DeMore (1982), is needed for a correct calculation of $\mathrm{O}$ (and $\mathrm{CO}$ ) production and loss rates. The 
two-dimensional model chemistry is now in excellent agreement with that given by Massie et al. (1983) for altitudes above 105 $\mathrm{km}$.

The model improvements introduced from Part $\mathrm{I}$, in radiative heating and cooling alone, have not had a significant impact on day-night temperature and density contrasts. In fact, only the global mean (and to a lesser extent nightside) temperatures are brought closer to Pioneer Venus values. Part I modifications are not sufficient by themselves to reproduce the observed thermospheric structure. The next section describes how eddy drag and diffusion parameters have been adjusted to alter the momentum balance, giving a weakened thermospheric circulation. A best match of calculated fields to Pioneer Venus observations is then obtained with these parameter changes.

\subsection{Alternative Parameterizations FOR TESTING}

There is ample evidence for turbulent or wave motion in the upper atmosphere of Venus (Seiff et al., 1980; Seiff, 1982; von Zahn et al., 1983). All these observations, however, are snapshots of vertical wave structure and have no information concerning horizontal scales or periods. The vertical wavelengths of these density and temperature perturbations have been observed to vary from 5 to $15 \mathrm{~km}$ in the altitude range of 120 to $140 \mathrm{~km}$. The effect of such waves on the small-scale mixing of densities and temperatures could be significant.

Eddy conduction, eddy or wave drag, and eddy diffusion have all been parameterized in other planetary models to mimic such turbulent processes. We specifically examine the role that these eddy processes serve in modifying composition, temperature, and winds in the two-dimensional model. All eddy-related parameterizations are eventually linked together with the use of a single reference eddy coefficient profile. This linkage maintains the aeronomical practice of connecting all turbulent terms by a single, prescribable coefficient based on mixing length theory. We do not attempt to make the large-scale fields and the eddy coefficient internally consistent. Rather, our model calculations are meant as a first step in the development of an internally consistent prescription that must evolve from a much better understanding of the sources of wave activity. The incorporation of these eddy processes presently means a loss of internal consistency for the two-dimensional model (von Zahn et al. 1983).

\section{(A) Eddy Diffusion and Conduction}

Previously developed one-dimensional composition models (von Zahn et al., 1980; Stewart et al., 1980; Massie et al., 1983; Shimazaki et al., 1984) indicate an upper limit to the magnitude of eddy diffusion needed in a Venus hydrodynamical model. We select a turbopause or cutoff value of the eddy coefficient $K_{t}$, the corresponding cutoff pressure level $Z_{\mathrm{t}}$, and a solar zenith angle variation (SZAF) as parameters for adjustment. These parameters are chosen for ease of comparison with previous onedimensional model work. The profile shape $K(z)=K_{\mathrm{t}}\left(p / p_{0}\right)^{-0.5}$, similar to that given by Lindzen (1971) and utilized in Part I, is likewise adopted here. Above 130-135 km ( $p<$ $p_{0}$ ), where molecular diffusion overwhelms eddy diffusion, $K(z)=K_{\mathrm{t}}$. Equation (2.2) incorporates a log-pressure form for the eddy diffusion parameterization similar to that used by Dickinson et al. (1984). We will show that the large-scale winds and small-scale mixing processes required for observed densities demand that $K(z)$ be some fraction of the one-dimensional model values used earlier. Eddy conduction, consistent with these reduced eddy coefficient values, is also incorporated using Eq. (2.3).

\section{(B) Wave Drag within the Horizontal Momentum Equation}

It has been shown that the heat and momentum budgets of the terrestrial meso- 
sphere cannot both be simultaneously satisfied without a strong zonal drag force (Lindzen, 1981; Holton, 1982; Holton, 1983; Matsuno, 1983). Such a frictional force is required to simulate the reversal of the latitudinal temperature gradient at the mesopause, with the lowest temperatures at the summer pole (due to adiabatic cooling from upwelling) and the warmest at the winter pole (due to adiabatic heating from subsidence). Wave drag weakens mean zonal winds near the mesopause despite the presence of a very strong differential radiative drive. The role of wave diffusion is seen to be secondary, acting only to smooth the vertical wind profile forced by wave drag (Holton, 1983).

The underlying mechanism responsible for such drag has long been argued to be wave-induced deceleration, resulting from the unstable breakdown of tides or internal gravity waves near the terrestrial turbopause. Gravity waves generated in the troposphere are assumed to attain sufficient amplitude in the mesosphere to produce layers with superadiabatic lapse rates. The convective overturning of such layers generates breaking waves which give rise to turbulence, and by limiting any further growth of wave amplitudes also causes a convergence of the vertical momentum flux. The resulting attenuation of the waves generates turbulent diffusion and also a net zonal drag force (Lindzen, 1981; Holton, 1982, 1983).

Early zonally symmetric models of the terrestrial meosphere were only partially successful in simulating the observed mean zonal winds. All such models (Schoeberl and Strobel, 1978; Holton and Wehrbein, 1980) showed that improved mean zonal winds could be obtained by specifying a height-dependent Rayleigh friction coefficient with a slow damping rate below 50 $\mathrm{km}$ and a fast damping rate near the mesosphere. It was difficult to justify these simple damping parameterizations on physical grounds. Nevertheless, they formed the basis for the current wave-drag models pat- terned after those of Lindzen (1981) and Holton (1982) which describe more selfconsistent methods for parameterizing the stress and diffusion due to gravity wave breaking in terms of the mean zonal wind, static stability, and specific wave parameters.

Our approach for the upper atmosphere of Venus has been guided by these terrestrial mesopause wave-drag parameterizations and the underlying physical mechanism of wave breaking. We assume that small-scale gravity waves are responsible for the observed wavelike variations in the altitude profiles of temperature and density (Seiff et al., 1980; Schubert, 1983) over $120-150 \mathrm{~km}$. The source of such waves could be convective activity at cloud heights near $65 \mathrm{~km}$ (Schubert, 1983). According to the "wave-breaking" scheme of Lindzen-Holton, these waves are assumed to grow in amplitude as $\exp (z / 2)$ up to breaking level $\left(Z_{t}\right)$ where overturning begins and eddy diffusion reaches a maximum $(130-135 \mathrm{~km})$. Deceleration of the subsolarto-antisolar winds is assumed to become significant at this breaking level. The turbulent eddy diffusion should attenuate any further growth of the waves above $Z_{t}$, in addition to the damping effect of molecular diffusion which is becoming dominant above the turbopause at $130-135 \mathrm{~km}$. The Lindzen-Holton scheme actually applies above a breaking level only. However, we assume a profile increasing with height for wave drag, which is consistent with a vertically propagating spectrum of breaking waves, each of which breaks at a different level.

Since virtually nothing is known about the actual spectral distribution of gravity waves in the Venus lower mesosphere and thermosphere (and little can be estimated without horizontal wind measurements from 60 to $140 \mathrm{~km}$ ), it seems impractical to use any parameterization for wave-induced acceleration more detailed than Rayleigh friction. That is, we assume drag is proportional to $u$ and not as in more detailed theo- 
ries to some power of $u-c$. Both Rayleigh friction and eddy viscosity terms are tested to crudely simulate the effects of wave breaking and drag on the mean flow. A Rayleigh friction scheme is first adopted, as used in previous terrestrial mesopause calculations (Schoeberl and Strobel, 1978; Holton and Wehrbein, 1980), to determine the approximate drag time scale needed to prescribe a weakened circulation consistent with observed atmospheric structure. The Rayleigh drag term in the equation of motion is of the form given by Eq. (2.1), where $u$ is subsolar to antisolar wind, $p$ is pressure, and $\lambda$ is the inverse of the drag time scale.

Initially, the drag is specified to be directly proportional to the horizontal wind and otherwise constant with altitude. However, the vertically propagating waves responsible for momentum flux convergence and drag are assumed to propagate upward through a wind system which selectively filters wave components that propagate in the same direction as the wind, and retains waves having phase speeds opposite to the prevailing wind. At some average level, the surviving waves are attenuated by breaking and retard the local winds. This suggests that Rayleigh drag varies with altitude. The wave-drag damping rate is next assumed to have a vertical profile similar to the eddy coefficient, i.e., $\lambda$ has the form

$$
\lambda=\lambda_{0} \frac{2}{1+\left(\frac{p}{p_{0}}\right)^{0.5}}
$$

where $\lambda_{0}$ and $p_{0}$ are adjustable parameters. Hence, $\lambda$ equals the constant $\lambda_{0}$ for $p=p_{0}$, and approaches zero for $p \gg p_{0}$. Such a profile is consistent with the growth factor for vertically propagating gravity waves (Lindzen, 1971). Winds are assumed to be largely uninhibited at low altitude, below a "breaking level." Above, the horizontal winds are reduced for an improved daynight contrast of calculated fields. Calculations of the next section assume for Eq.
(3.1) that $\lambda_{0}=1.2 \times 10^{-4} \mathrm{sec}^{-1}$ and $p_{0}=7 \times$ $10^{-4} \mu$ bar.

Finally, a more satisfying and internally consistent parameterization is attempted which makes use of the vertical eddy coefficient profile. Molecular viscosity is altered to include a component simulating the influence of small-scale mixing (see Dickinson et al., 1984):

$$
\mu_{\mathrm{e}}=\rho K(z)
$$

This parameterization, based on an eddy coefficient which grows with height with the vertical dependence described earlier, permits drag to increase with altitude also. Eddy viscosity is linked to the other eddy parameterizations through the use of a common eddy coefficient profile.

To proceed with model sensitivity studies, we first develop a Rayleigh friction parameterization giving good model agreement between calculated and observed composition and temperature fields. Vertical eddy diffusion of composition is added afterward for a fine-tuning of densities, particularly on the nightside. Finally, optimal parameter adjustments are specified combining consistent eddy viscosity and diffusion in concert with reduced Rayleigh friction to give a final reference model calculation. Our goal is to confine the influence of eddy processes to the minimum required to augment the role of the large-scale winds in the initial reference model.

A least-squares fitting procedure (similar to Gerard et al., 1981) is used to quantify the degree of fit of the calculated two-dimensional fields to the reference empirical model values of Hedin et al. (1983) [VTS3] and Keating et al. (1984) [HSYM], for solar maximum $(F 10.7=200)$ and solar medium $(F 10.7=150)$ conditions, respectively. Our final reference model calculation provides a reasonable description of the mean thermospheric structure and circulation, midway between the empirical model fields described above. The model also predicts densities and temperatures below $140 \mathrm{~km}$, 

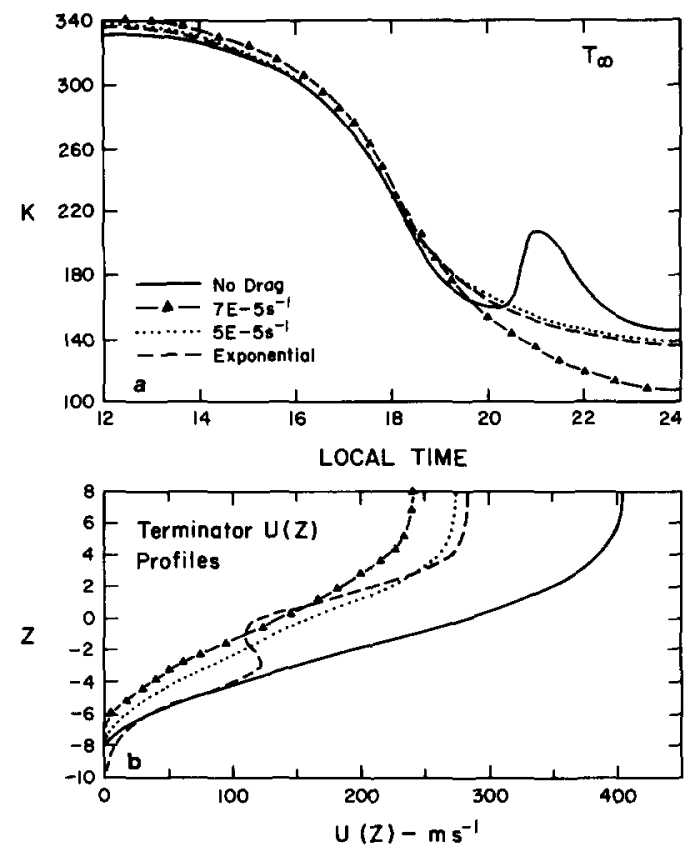

FIG. 4. Venus two-dimensional model response to viscous drag tests: (a) exospheric temperatures and (b) terminator wind profiles. Various drag time scales are examined to determine the general response by wind and exospheric temperatures. Notice that global mean temperatures are largely unchanged by different drag rates, hence different winds.

outside the region of in situ global observations.

\subsection{Results of Sensitivity Studies}

\section{(A) General Response to Eddy Processes}

We first examine qualitatively the way eddy diffusion, eddy conduction, and wave-drag combine to influence the composition, temperature, and wind fields. We look for changes that will give increased dayside light gases $(\mathrm{O}$ and $\mathrm{CO})$, depleted nightside light gases, and cooler nightside temperatures compared to the DRM values.

Several different time scales for wave drag are tested using Rayleigh friction. These tests show what magnitude of drag is necessary within the momentum equation for our winds to approximate those estimated by Mayr et al. (1980). For this analy- sis, we assume that wave drag is linearly proportional to horizontal velocity but otherwise constant with altitude. Damping rates ranging from $5 \times 10^{-5}$ to $2 \times 10^{-4}$ $\mathrm{sec}^{-1}$ are considered. The resulting terminator winds for rates of $5 \times 10^{-5}$ and $7 \times$ $10^{-5} \mathrm{sec}^{-1}$ are selected for comparison in Fig. 4b, starting with output from the case 1 two-dimensional run of Section 3.1. The exponential profile shown corresponds to a vertical profile for drag according to Eq. (3.1), to be discussed below. Notice that terminator winds are reduced by nearly $50 \%$ to $240 \mathrm{~m} \mathrm{sec}^{-1}$ at exospheric heights.

This reduction of winds is accompanied by a very striking modification of exospheric temperatures. Dayside heat is isolated from the nightside with slower winds, giving colder nightside temperatures. Figure $4 a$ illustrates that the day-night temperature contrast is enhanced by wave drag; dayside temperatures increase and nightside temperatures decrease. The nightside warming feature (due to compressional heating) in the DRM exospheric temperatures near $\mathrm{LT}=21 \mathrm{hr}$ is eliminated by drag (for a rate of $5 \times 10^{-5} \mathrm{sec}^{-1}$ or larger). Nightside temperatures drop to values as low as $110^{\circ} \mathrm{K}$ with a drag rate of $7 \times 10^{-5}$ $\mathrm{sec}^{-1}$. Wave drag does not, however, greatly alter global mean temperatures, indicating that the corrected final reference model of Part I is still a valid description of global mean balances.

Figure 2 shows that with these slower winds, day and night mixing ratios are closer to those observed, particularly for $\lambda$ $=1 \times 10^{-4} \mathrm{sec}^{-1}$. Dayside fractional $\mathrm{O}$ increases significantly toward VTS3 empirical model values, while nightside $\mathrm{O}$ is only slightly reduced. More of the photodissociated $\mathrm{O}$ and $\mathrm{CO}$ is retained on the dayside. This trend suggests that an observed distribution of composition can be achieved provided that sufficient eddy drag is used. Eddy diffusion may also be required to provide a further reduction of nightside densities.

The case for $\lambda=1 \times 10^{-4} \mathrm{sec}^{-1}$, constant 
drag with altitude, is now combined with eddy diffusion to examine how large-scale winds and eddy diffusion simultaneously control densities. A series of eddy diffusion tests is conducted with $K_{\mathrm{t}}$ ranging from 200 to $1500 \mathrm{~m}^{2} \mathrm{sec}^{-1}$ and $Z_{\mathrm{t}}$ from -4 to 1 . We find that eddy diffusion and correspondingly $K(z)$ must be roughly a factor of 2 stronger on the nightside than the dayside, regardless of which $Z_{t}$ is chosen. Since the large-scale winds very effectively control upper dayside composition (Sect. 3.3), eddy diffusion cannot be of help in maintaining the observed dayside $\mathrm{O}$ and $\mathrm{CO}$ densities. Thus a dayside "threshold" value of $K(z)$ is obtained, for which eddy diffusion has an insignificant impact on dayside densities. For a mean turbopause near $130 \mathrm{~km}$, the observed dayside composition requires that $K_{\mathrm{t}}$ be approximately $500 \mathrm{~m}^{2}$ $\mathrm{sec}^{-1}$ or less. Nightside densities are improved by stronger eddy diffusion, that is $K_{\mathrm{t}}=1000 \mathrm{~m}^{2} \mathrm{sec}^{-1}$. Greater eddy diffusion on the nightside is consistent with a more dynamically unstable nightside thermosphere, where pronounced wave signatures have been observed. The failure of the reduced large-scale winds to simultaneously provide observed dayside and nightside densities is rectified by such day-night differences in eddy diffusion. The maximum coefficient for global mean eddy diffusion $K_{\mathrm{t}}$ is approximately $750 \mathrm{~m}^{2} \mathrm{sec}^{-1}$.

The requirement that $K_{\mathrm{t}}<1000 \mathrm{~m}^{2} \mathrm{sec}^{-1}$ on the dayside is less than needed to give major dayside eddy cooling $\left(K_{\mathrm{t}}>4000 \mathrm{~m}^{2}\right.$ $\mathrm{sec}^{-1}$ was required in Part I for a $10 \%$ EUV efficiency with weak $15-\mu \mathrm{m}$ cooling). Furthermore, eddy cooling cannot act strongly on the nightside simultaneously with a weakened circulation without giving too cold nightside temperatures. Hence, eddy conduction, using coefficients that do not exceed those needed by diffusion, can provide only a minor contribution to the total cooling required for calculating observed dayside temperatures, as argued in Part $\mathrm{I}$. Corrected thermal balance parameters from Fig. 4 of Part I have been examined in the two-dimensional model in light of this re- striction $\left(K_{\mathrm{t}}<1000 \mathrm{~m}^{2} \mathrm{sec}^{-1}\right.$ and $K_{\mathrm{CO}_{2}-\mathrm{O}}<$ $\left.9 \times 10^{-13} \mathrm{~cm}^{3} \mathrm{sec}^{-1}\right)$. Any slight improvement in temperatures above $150 \mathrm{~km}$ is offset by cooler temperatures around 130-140 $\mathrm{km}$ that depart significantly from observations (see Fig. 3 of Part I). Eddy conduction is neglected for further calculations, and instead strong $15-\mu \mathrm{m}$ cooling is used to balance plausible EUV heating in conjunction with optimally adjusted eddy drag and diffusion parameters to provide a final reference model calculation.

\section{(B) Detailed Sensitivity Studies}

Our primary objective in choosing parameters for Eq. (3.1) is to devise a drag profile that gives a smooth transition from low- to high-altitude wind regimes and a best match of the calculated fields to those given by the PV observations. The calculated circulation should simultaneously maintain observed dayside densities and a reasonable nightside column of observed temperatures and composition. Eddy diffusion is adjusted, as needed, to provide slight improvements to nightside densities.

First, we adjust the $p_{0}$ and $\lambda_{0}$ parameters in Eq. (3.1). This $p_{0}$ level might be considered a transition region above which molecular diffusion processes damp any further growth of gravity wave perturbations. Figure 5 illustrates a family of terminator wind profiles that result from these adjustments. Generally, as $p_{0}$ is raised, corresponding to a higher breaking level, low-altitude winds are enhanced, and the day-night temperature contrast is weakened below $z=-4$ $(120 \mathrm{~km})$. Upper level winds are calculated to be less than $230 \mathrm{~m} \mathrm{sec}^{-1}$, in accord with the qualitative studies of the previous section. The pressure dependence of the wavedrag term effectively smooths out the transition from low- to high-altitude winds by permitting the (negative) momentum to be deposited more gradually over a wider altitude region. A best drag profile is adopted with $p_{0}=7 \times 10^{-4} \mu$ bar and $\lambda_{0}=1.2 \times 10^{-4}$ $\mathrm{sec}^{-1}$, giving winds $<230 \mathrm{~m} \mathrm{sec}^{-1}$.

The improvements in global tempera- 


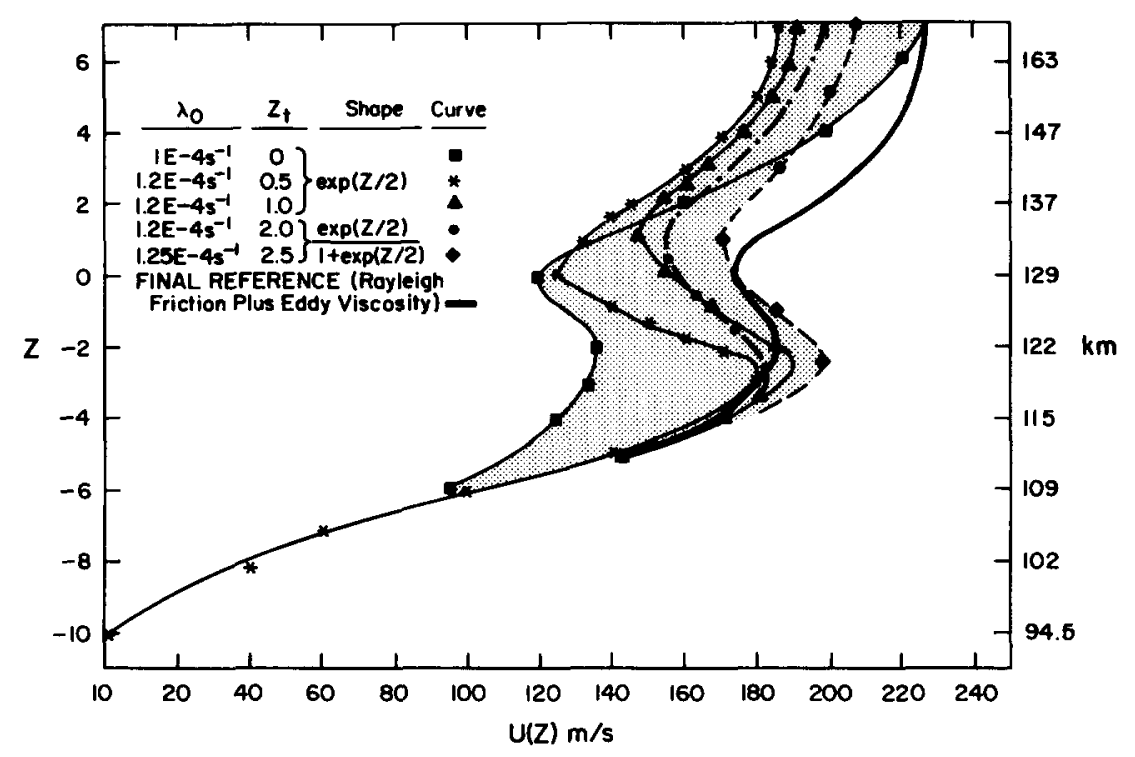

FIG. 5. Family of terminator horizontal wind profiles. Various vertical profile parameterizations for wave drag are tested and the corresponding terminator winds are presented. With decreasing $p_{0}$ (rising breaking level), lower altitude winds are stronger. Maximum horizontal winds are required to be $<230$ $\mathrm{m} \mathrm{sec} \mathbf{s}^{-1}$.

tures and composition are striking. Figure 6 shows nightside exospheric temperatures for the best drag profile rising $10-15^{\circ}$ over the previous constant drag case $\left(\lambda_{0}=10^{-4}\right.$ $\sec ^{-1}$ ), now giving $113^{\circ} \mathrm{K}$ at midnight. At the same time, global mean and dayside exospheric temperatures rise toward VTS3 values. Dayside composition does not change appreciably, since the largest increase in winds occurs at altitudes below $135 \mathrm{~km}$, where the transported species $\mathrm{O}$ and $\mathrm{CO}$ are minor compared to $\mathrm{CO}_{2}$. It ap-

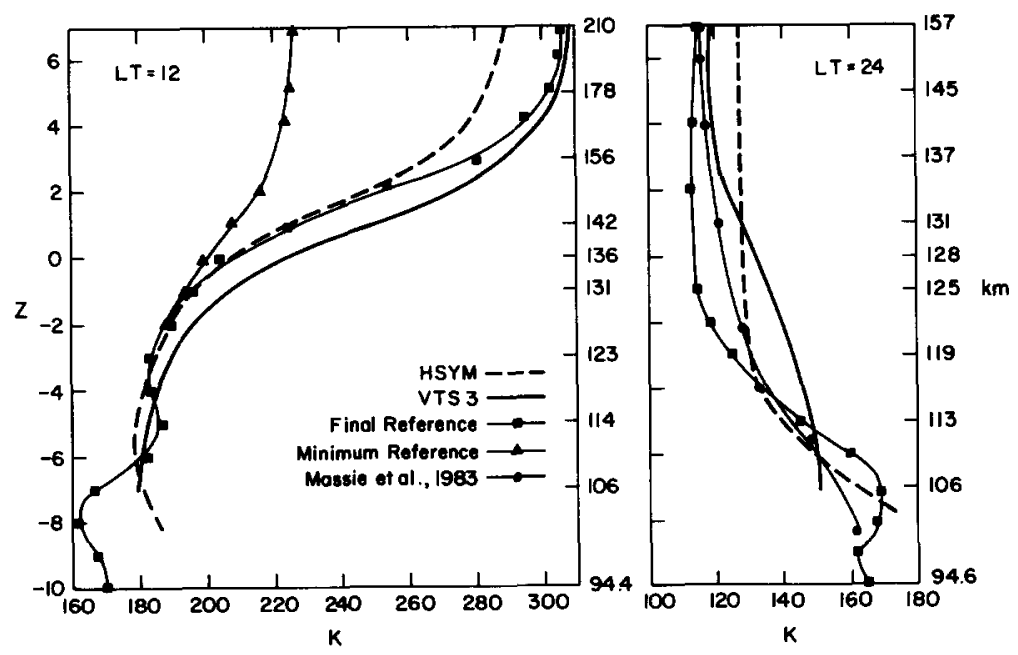

Fig. 6. Venus two-dimensional model temperatures: final reference. Calculated vertical temperature profiles at noon and midnight are presented and compared to the corresponding empirical model profiles of Hedin et al. (1983) [VTS3] and Keating et al. (1984) [HSYM]. Nightside temperatures are also compared to the midnight profile of Massie et al. (1983). 
pears that largely uninhibited low-altitude winds are crucial to the maintenance of an expanded and warmer nightside column. Horizontal winds at $115 \mathrm{~km}$ approach $140 \mathrm{~m}$ $\mathrm{sec}^{-1}$ at the terminator, in near agreement with the $130 \mathrm{~m} \mathrm{sec}^{-1}$ Earth-based absolute wind velocities measured by Betz (1982), using high-resolution heterodyne spectroscopic techniques. These low-altitude winds permit thermal advection and adiabatic compression to redistribute heat about the planet, giving a very small temperature variation over solar zenith angle. Temperatures at $100 \mathrm{~km}$ for the best drag profile approach $160-170^{\circ} \mathrm{K}$ globally, in contrast to the constant drag profile calculations which gave lower boundary values of 190 and $150^{\circ} \mathrm{K}$ for day and night, respectively. At lower levels, winds are somewhat faster than possible for constant drag, enabling thermal advection and adiabatic compression to contribute to the warmer nightside thermospheric temperatures calculated.

It appears that no mechanism other than drag is capable of retaining $\mathrm{O}$ and $\mathrm{CO}$ on the dayside at levels close to those observed. The drag necessary to do this regulates the ability of these same large-scale winds to transport $\mathrm{O}$ and $\mathrm{CO}$ to the nightside and to provide an adequate heat source to sufficiently maintain the nightside temperatures. A smoother wind structure helps to improve the day-night contrasts that result. This Rayleigh friction prescription provides a first estimate for the magnitude of wave drag needed to reproduce the observed day-night distribution of composition and temperatures.

Eddy viscosity can provide but a small fraction of the drag needed to diminish winds in our simulation. It is prescribed to be equal to the product of mass density and the vertical eddy coefficient for an internal consistency among all eddy-related terms [see Eq. (3.2)]. This eddy viscosity profile again allows uninhibited low-altitude winds to blow. However, this drag alone is inadequate to weaken upper level winds suffi- ciently to derive observed day-night density and temperature contrasts. A strong feedback in fact exists among dayside atomic $\mathrm{O}$, temperatures, and winds due to the strong $15-\mu \mathrm{m}$ cooling used. Depleted O resulting from strong winds causes temperatures to rise, driving still stronger winds. Maximum terminator winds, with drag only by eddy viscosity, are roughly $400 \mathrm{~m} \mathrm{sec}^{-1}$, nearly $180 \mathrm{~m} \mathrm{sec}^{-1}$ faster than found previously from the Rayleigh friction calculation. The relatively minor reduction of global winds by eddy viscosity $(<50 \mathrm{~m}$ $\mathrm{sec}^{-1}$ ) gives severely depleted dayside densities of $\mathrm{O}$ and $\mathrm{CO}$, with corresponding nightside enhancements, much like our initial reference model calculations.

We now combine a vertically varying Rayleigh friction $\left(\lambda_{0}=8.8 \times 10^{-5} \mathrm{sec}^{-1}, p_{0}\right.$ $=1.1 \times 10^{-3} \mu$ bar) with eddy viscosity, using a $K(z)$ profile which is the average of the day-night value used for diffusion, to provide the needed wave drag in a final reference model calculation. This combination smooths the horizontal wind structure, enabling improved nightside densities and temperatures to be derived while maintaining the dayside $\mathrm{O}$ and $\mathrm{CO}$ densities and temperatures observed. Eddy diffusion and viscosity are now linked through a prescribed eddy coefficient. Final reference model parameters are summarized in Table I.

\subsection{Final Reference Model Results AND DiagnOSTICS}

Wave drag, given by a combination of Rayleigh friction and eddy viscosity, is the primary means used to obtain day-to-night temperature variations and $\mathrm{O}$ and $\mathrm{CO}$ concentrations similar to those observed by $\mathrm{Pi}$ oneer Venus. Figure 6 illustrates noon and midnight profiles of calculated temperatures for comparison with empirical model values of Hedin et al. (1983) and Keating et al. (1984). The calculated dayside temperature profile corresponds closely to VTS3 temperatures above $105 \mathrm{~km}$, and gives a dayside exospheric value of $304^{\circ} \mathrm{K}$. Night- 
TABLE I

Final Reference Model Parameters

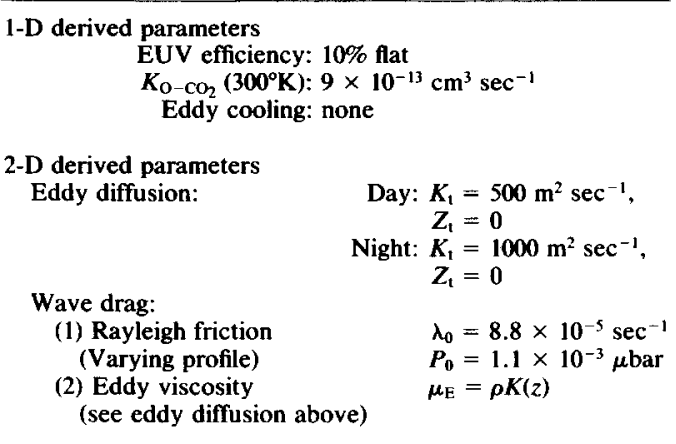

side temperatures also approach empirical model values, resulting in the expansion of the entire nightside column. The midnight exospheric temperature is now $113^{\circ} \mathrm{K}$, which is quite close to VTS3 and Massie et al. (1983) values of 118 and $115^{\circ} \mathrm{K}$, respectively. The intermediate temperatures $(-4$ $<z<2$ ), however, are approximately $10^{\circ} \mathrm{K}$ cooler than observed. Lower boundary temperatures, particularly on the dayside, are also $10-15^{\circ} \mathrm{K}$ cooler than Orbiter Infrared Radiometer (OIR) observations. The heating mechanisms at altitudes below 105 $\mathrm{km}$ have not been explicitly examined in this study.

Dayside fractional $O$ densities are now calculated to be much closer to observed values than the previous DRM. The abundance ratio $\left(\mathrm{O} / \mathrm{CO}_{2}\right)$ at the altitude of the dayside ionospheric peak $(140 \mathrm{~km})$ is commonly quoted to compare model and observed $\mathrm{O}$ densities. This value for our final reference calculation is 0.14 , which is nearly 2.5 times that given previously by DRM, and compares quite well with the observed value of 0.17 from the Bus Neutral Mass Spectrometer measurements of von Zahn et al. (1980). Still slower large-scale winds would help to further increase the calculated dayside fractional $O$. Nightside fractional $\mathrm{CO}$ densities are also somewhat low $(0.06$ versus 0.09 at $140 \mathrm{~km}$ at midnight), and nightside temperatures are slightly low. These latter features would be improved by stronger winds.

The nightside density at a given altitude is highly dependent on the reference lower boundary altitude at $z=-10$ (or $p=0.1$ mbar). Presently, values ranging from 94.4 to $94.6 \mathrm{~km}$ from day to night are specified in accord with the one-dimensional final reference model calculations of Part I. These values are generally consistent with altitude scales available from the probe data of Seiff and Kirk (1982), the empirical models of Hedin et al. (1983) and Keating et al. (1984), and the Massie et al. (1983) onedimensional composition model. A few tenths of a kilometer adjustment can result in significant differences in nightside densities, making precise comparisons of model results with empirical model atmospheres difficult.

We examine the thermal, momentum, and compositional balances present for this optimal final reference case. Figure 7 shows heating and cooling balances at $\theta=60$ and $120^{\circ}$, representing dayside and nightside conditions. As in the DRM, dayside upper level EUV heating $(z>1)$ approximately balances downward molecular conduction and $15-\mu \mathrm{m}$ cooling, while low-altitude infrared heating $(z<0)$ is primarily offset by 15 $\mu \mathrm{m}$ cooling. This reference case EUV heating and $15-\mu \mathrm{m}$ cooling are roughly a factor of 2.25 greater than the previous dayside

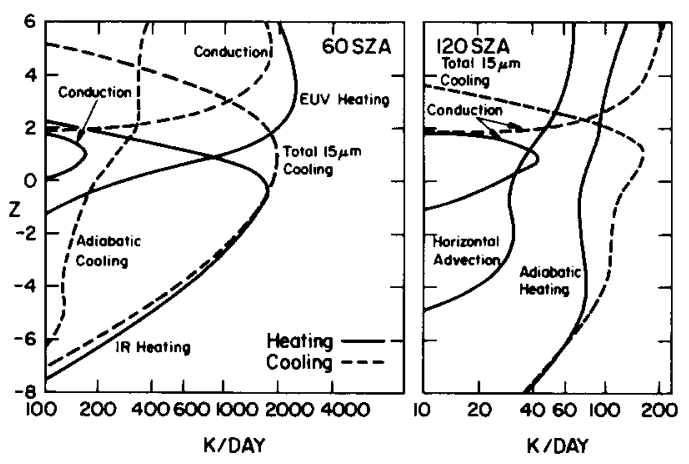

FIG. 7. Heating and cooling rates: $\theta=60^{\circ}$ and $120^{\circ}$. Heat balance for mean dayside and nightside conditions is presented for our final reference two-dimensional model calculation. Units: ${ }^{\circ} \mathrm{K}$ per Earth day. 


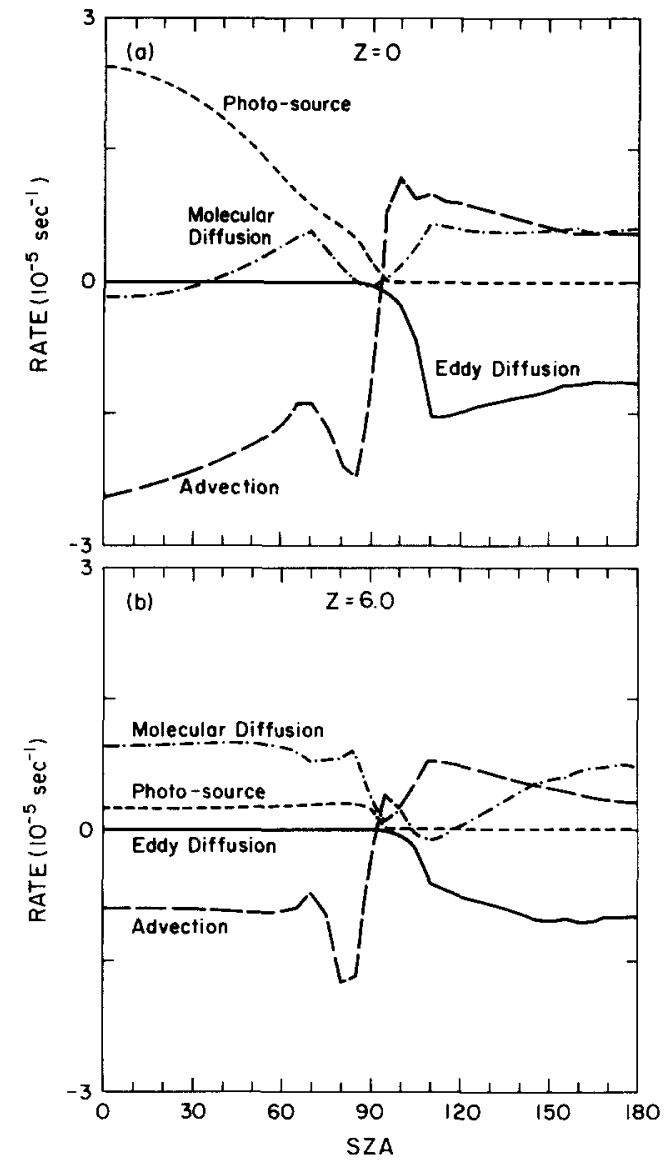

FIG. 8. Composition balance for atomic $\mathrm{O}$ equation. Term balances at two pressure levels are presented as rates in units of $\sec ^{-1}$.

DRM model values at $145 \mathrm{~km}$ (or $z=2$ ), resulting primarily from larger solar fluxes and more efficient EUV heating which are balanced by $15-\mu \mathrm{m}$ cooling. The nightside balance is primarily between adiabatic compressional heating and $15-\mu \mathrm{m}$ cooling, with additional high-altitude cooling resulting from conduction. The nightside adiabatic heating is less with slower winds; that is, nightside compressional heating is reduced to a third of its DRM level above $140 \mathrm{~km}$ (or $z=3$ ). Likewise, horizontal thermal advection is reduced by almost half along with 15 $\mu \mathrm{m}$ cooling on the nightside. The smoother and stronger low-altitude wind profile provides increased warming of the lower night- side thermosphere not otherwise possible with constant drag. In short, we demonstrate that the nightside temperatures above $140 \mathrm{~km}$ require a relatively weak circulation. Below $140 \mathrm{~km}$, nightside adiabatic heating largely balances $15-\mu \mathrm{m}$ cooling.

A weak circulation is not only important to a proper nightside thermal balance, but also to a reasonable distribution of composition about the planet. Figures 8 and 9 show term balances for fractional $\mathrm{O}$ and $u$ fields over all solar zenith angles for specific pressure levels. Comparison with previous DRM balance plots reveals the significant role eddy diffusion now has in determining nightside $\mathrm{O}$ densities $(z>0)$. The transport source terms for $\mathrm{O}$ (and $\mathrm{CO}$ )
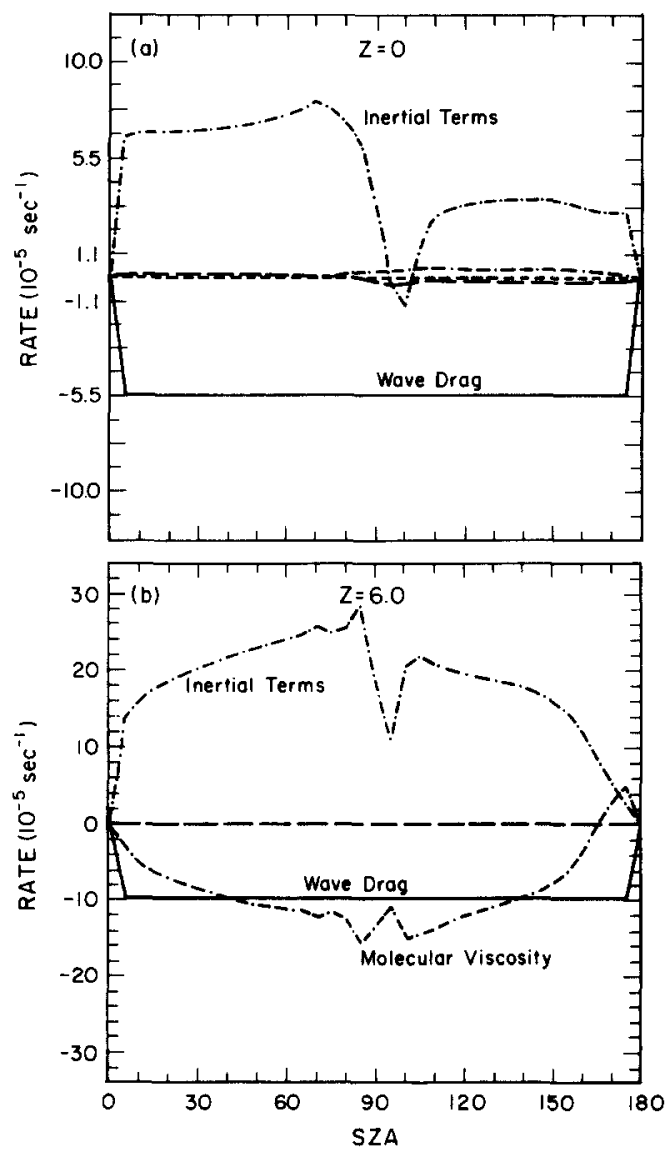

FIG. 9. Balance within horizontal momentum equation. Term balances at two pressure levels are presented as rates in units of $\mathrm{sec}^{-1}$. 
TABLE II

Model and Empirical Data Set Comparisons AT $150 \mathrm{~km}$

\begin{tabular}{|c|c|c|c|c|c|}
\hline \multicolumn{6}{|c|}{ Noon, $\mathrm{LT}=12 \mathrm{hr}$} \\
\hline & VTS3a $^{a}$ & VTS3/2D & HSYM $^{b}$ & HSYM/2D & $2 \mathrm{D}$ \\
\hline$n_{\text {TOT }}$ & $1.8(10)$ & 1.24 & $1.75(10)$ & 1.21 & $1.45(10)$ \\
\hline o & $5.4(9)$ & 1.80 & $4.0(9)$ & 1.33 & $3.0(9)$ \\
\hline $\mathrm{CO}$ & $3.2(9)$ & 1.36 & $2.34(9)$ & 0.99 & $2.36(9)$ \\
\hline $\mathrm{CO}_{2}$ & 8.1 (9) & 1.02 & $9.81(9)$ & 1.24 & $7.94(9)$ \\
\hline $\mathbf{N}_{2}$ & $1.3(9)$ & 1.09 & $1.32(9)$ & 1.11 & $1.19(9)$ \\
\hline$T$ & 273.0 & 1.05 & 246.5 & 0.95 & 260.6 \\
\hline \multicolumn{6}{|c|}{ Midnight, LT $=24 \mathrm{hr}$} \\
\hline & VTS3 & VTS3/2D & HSYM & HSYM/2D & $2 \mathrm{D}$ \\
\hline$n_{\text {TOT }}$ & $8.8(8)$ & 1.0 & $1.08(9)$ & 1.22 & $8.83(8)$ \\
\hline 0 & $7.04(8)$ & 0.93 & $8.51(8)$ & 1.12 & $7.60(8)$ \\
\hline $\mathrm{CO}$ & $5.72(7)$ & 1.86 & $7.24(7)$ & 2.36 & $3.07(7)$ \\
\hline $\mathrm{CO}_{2}$ & $6.25(7)$ & 1.19 & $7.10(7)$ & 1.35 & $5.24(7)$ \\
\hline $\mathbf{N}_{2}$ & $4.14(7)$ & 1.05 & $5.91(7)$ & 1.50 & $3.93(7)$ \\
\hline$T$ & 118.0 & 1.04 & 127.4 & 1.13 & 113.0 \\
\hline
\end{tabular}

Note. Values in parentheses are the powers of 10 by which the primary tabular entry is to be multiplied.

a VTS3, F10.7 $\Rightarrow 206 \times 10^{-22} \mathrm{~W} \mathrm{~m}^{-2} \mathrm{~Hz}^{-1}$

${ }^{b}$ HSYM, $F 10.7 \approx 150 \times 10^{-22} \mathrm{~W} \mathrm{~m}^{-2} \mathrm{~Hz}^{-1}$.

are also smaller, giving additional cause for diminished nightside $\mathrm{O}$ (and $\mathrm{CO}$ ). This redistribution of gases about the planet is the result of wave drag, which now provides a great deal of control over the momentum balance, particularly for $z>0$.

Table II compares number densities $(\mathrm{O}$, $\mathrm{CO}, \mathrm{CO}_{2}, \mathrm{~N}_{2}$, total) and temperatures at 150 $\mathrm{km}$ for our final reference model calculation with the reference empirical model atmospheres. This level is chosen since it represents the lower boundary (mean periapsis altitude) of many of the orbiter-generated data sets. An examination of number densities provides a useful check of the character of an entire vertical column, particularly for the nightside where integrated temperature structure is of greatest interest. The $\mathrm{N}_{2}$ mass mixing ratio is specified according to the Hedin et al. (1983) empirical model within our two-dimensional model. Its precise number density depends on the thermal structure and total number density calculated. We compare dayside and nightside profiles with observations at LT $=12$ and 24. This comparison reflects the strength of our two-dimensional calculation in repro- ducing mean day-night contrasts, for which a nearly monotonic decrease of temperature is calculated from noon to midnight.

Our mean model calculation generally provides fields that fall within the range of empirical model densities and temperatures specified by Hedin et al. (1983) and Keating et al. (1984). The calculated dayside densities and temperatures match observations within approximately $30 \%$, with the largest discrepancies seen in $\mathrm{O}$ and $\mathrm{CO}_{2}$ densities. Nightside model fields depart further from empirical model values. We find it difficult to calculate good dayside composition and adequate nightside densities and temperatures simultaneously. Notice that dayside $\mathrm{O}$ is slightly less than the observed levels, suggesting the need for slower winds. However, nightside densities (in particular $\mathrm{CO}$ ) are too small, indicating a collapsed altitude scale. These nightside densities could be improved with a faster wind structure for which warmer nightside temperatures could be maintained, but at the expense of poorer dayside densities and temperatures.

Contours of the $T, u, w$, and fractional $O$ and $\mathrm{CO}$ fields for the final reference twodimensional model calculation are displayed on an altitude scale in Figs. 10b through $f$, respectively. The mapping of altitude onto log-pressure coordinates (used in our model integrations) is displayed in Fig. 10a. The sharp contour at $z=7$ represents the upper boundary of our model, an approximate exobase. The calculated distribution of densities and temperatures reproduces the sharp terminator transition seen in the Pioneer Venus observations. Warm dayside temperatures are maintained, as well as very cold nightside "cryosphere" temperatures. Notice that thermospheric temperatures and mixing ratios near the ionospheric peak $(140 \mathrm{~km})$ are almost independent of local time on the dayside. This is a striking difference to the diurnal behavior of the terrestrial thermosphere. We attribute this to the increased importance of our $15-\mu \mathrm{m}$ cooling in 

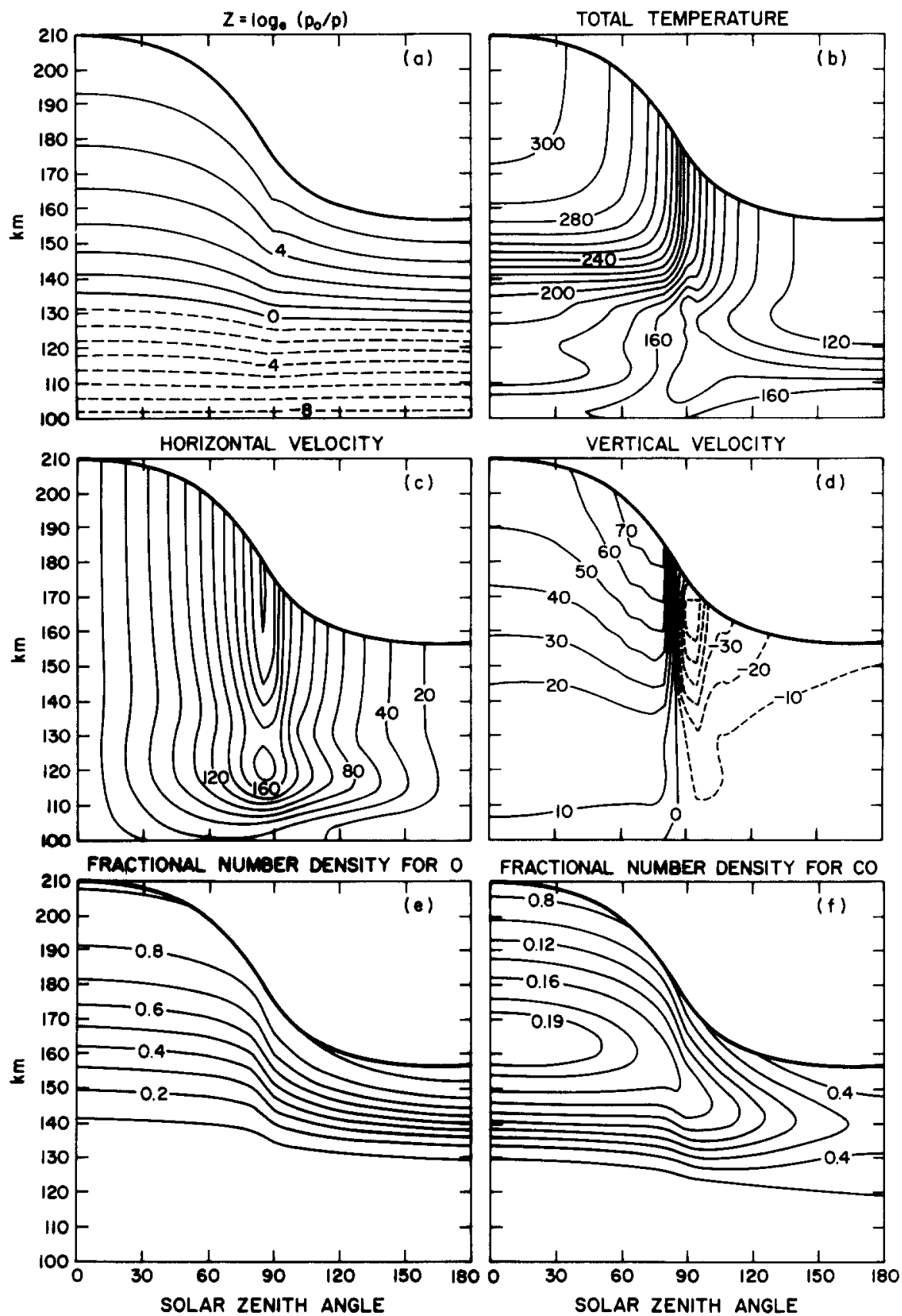

Fig. 10. Solar maximum final reference model contour plots. All plots are presented on an altitude scale for convenience. The sharp upper level cufoff $(z=7)$ corresponds to the top boundary of our model calculations. (a) $z=\log _{\mathrm{e}}\left(p_{0} / p\right)$; (b) total temperature in ${ }^{\circ} \mathrm{K}$; (c) horizontal velocity in $\mathrm{m} \mathrm{sec}^{-1}$; (d) vertical velocity in $\mathrm{cm} \mathrm{sec}^{-1}$; (e) fractional number density for $\mathrm{O}$; (f) fractional number density for $\mathrm{CO}$. 
balancing EUV heating. $\mathrm{CO}_{2} 15-\mu \mathrm{m}$ cooling serves to reduce the local time variation of temperatures from that which would occur if a balance were present between peak EUV heating and molecular conduction (the case for the Earth).

This calculated weak dayside temperature variation near the peak of EUV heating is not inconsistent with the observed ionospheric peak at $140 \mathrm{~km}$ for zenith angles less than $70^{\circ}$ (Cravens et al., 1981). Figure 10a shows that constant pressure surfaces in the vicinity of $140 \mathrm{~km}$ drop in height from $\theta$ $=0$ to $70^{\circ}$, which is consistent with the decreasing densities and near-constant temperatures. According to Chapman theory, the ionospheric peak will remain at a constant height over the dayside if the neutral density decreases at a rate sufficient to compensate for the increase in the length of the path due to the angular effect (Cravens et al., 1981). This condition holds for Venus and our two-dimensional model calculations up to $\theta=70^{\circ}$. We estimate a dayside ionospheric peak at roughly $139 \mathrm{~km}$.

Table III lists calculated fields at selected solar zenith angles for reference. The symmetric two-dimensional model is largely successful in simulating observed mean day-night contrasts. However, we cannot discuss with our symmetric model the asymmetric characteristics of the Venus thermosphere observed and inferred from Pioneer Venus. Retrograde zonal winds must be incorporated in a fully three-dimensional model to address these observations.

\subsection{Further Model Applications}

Additional tests provide a further validation of the model's ability to reliably simulate the observed Venusian thermospheric fields under a wide range of conditions. We first explore the two-dimensional model response of the $T, u, w$, and fractional $\mathrm{O}$ and $\mathrm{CO}$ fields to variations in solar flux over a solar cycle. The primary solar cycle changes occur in the EUV fluxes shortward of $1050 \AA$. Most other inputs and eddy or

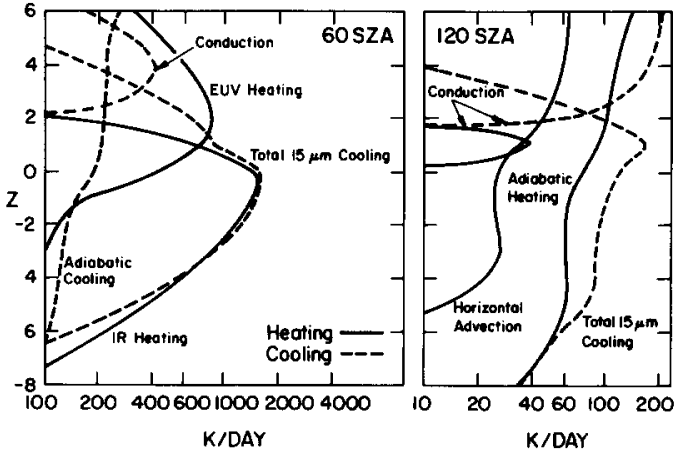

Fig. 11. Heating and cooling rates: $\theta=60^{\circ}$ and $120^{\circ}$. Heat balance for mean dayside and nightside conditions is presented for our minimum reference two-dimensional model calculation. Units: ${ }^{\circ} \mathrm{K}$ per Earth day.

cooling parameterizations are retained as before. Rayleigh friction makes use of a breaking level, $p_{0}=5 \times 10^{-3} \mu \mathrm{bar}$, which is lower than for solar maximum for a realistic horizontal wind structure. Heroux and Hinteregger (1978) fluxes (identical to Part I solar minimum values) are now adopted for the specific examination of solar minimum conditions $\left(F 10.7=74 \times 10^{-22} \mathrm{~W} \mathrm{~m}^{-2}\right.$ $\mathrm{Hz}^{-1}$ ). The final two-dimensional reference model above serves as the solar maximum standard for comparison.

Figure 11 illustrates heating and cooling balances at $\theta=60$ and $120^{\circ}$. The incorporation of solar minimum fluxes results in a reduction of EUV heating by approximately a factor of 3 . IR heating, important below $z=0$, remains virtually unchanged. Corresponding $15-\mu \mathrm{m}$ cooling is also reduced by a third at the level of peak EUV heating. Figure 12 illustrates steady-state contours of log-pressure (12a), temperature (12b), horizontal wind (12c), and fractional $\mathrm{O}$ (12d) fields calculated for this solar minimum test. Notice that dayside exospheric temperatures approach $225^{\circ} \mathrm{K}$ at $\mathrm{LT}=12$, which is a reduction of nearly $70^{\circ} \mathrm{K}$. Dayside temperatures at lower levels are also reduced. These newly calculated temperatures are somewhat low, yet the values fall within the range of exospheric temperatures obtained from Mariner 5 and $10 \mathrm{Ly}$ - 
TABLE III

Calculated Model Fields at Selected Solar Zenith Angles

\begin{tabular}{|c|c|c|c|c|c|c|c|}
\hline$Z$ & $\begin{array}{l}\text { Height } \\
(\mathrm{km})\end{array}$ & $\begin{array}{l}\text { Scale height } \\
(\mathrm{km})\end{array}$ & $\begin{array}{l}\text { Total number } \\
\text { density } \\
\left(\mathrm{cm}^{-3}\right)\end{array}$ & $\begin{array}{l}\text { Relative number } \\
\text { density } \\
\text { (O) }\end{array}$ & $\begin{array}{l}\text { Relative number } \\
\text { density } \\
\text { (CO) }\end{array}$ & $\begin{array}{c}T \\
\left({ }^{\circ} \mathrm{K}\right)\end{array}$ & $\begin{array}{c}u \text { or } w^{a} \\
\left.(\mathrm{~m} \mathrm{sec})^{-1}\right)\end{array}$ \\
\hline \multicolumn{8}{|c|}{ Solar zenith angle $=0^{\circ}$} \\
\hline-8.55 & 100 & 3.73 & $1.14(15)$ & .0001 & .0001 & 163.5 & 0.071 \\
\hline-7.20 & 105 & 3.78 & $2.95(14)$ & .0003 & .0003 & 164.9 & 0.091 \\
\hline-5.95 & 110 & 4.18 & $7.59(13)$ & .0007 & .0008 & 182.6 & 0.118 \\
\hline-4.77 & 115 & 4.28 & $2.30(13)$ & .0019 & .0020 & 186.7 & 0.137 \\
\hline-3.59 & 120 & 4.21 & $7.21(12)$ & .0046 & .0047 & 182.8 & 0.142 \\
\hline-2.42 & 125 & 4.34 & $2.18(12)$ & .0096 & .0100 & 186.9 & 0.146 \\
\hline-1.30 & 130 & 4.58 & $6.83(11)$ & .0191 & .0208 & 194.9 & 0.151 \\
\hline-0.24 & 135 & 4.86 & $2.31(11)$ & .0435 & .0509 & 200.0 & 0.154 \\
\hline 0.72 & 140 & 5.51 & $8.15(10)$ & .0856 & .0942 & 216.1 & 0.172 \\
\hline 1.55 & 145 & 6.45 & $3.21(10)$ & .1407 & .1333 & 238.5 & 0.202 \\
\hline 2.26 & 150 & 7.52 & $1.45(10)$ & .2066 & .1633 & 260.6 & 0.237 \\
\hline 2.88 & 155 & 8.58 & $7.38(9)$ & .2820 & .1841 & 277.0 & 0.272 \\
\hline 3.42 & 160 & 9.63 & $4.13(9)$ & .3642 & .1959 & 287.8 & 0.307 \\
\hline 3.90 & 165 & 10.68 & $2.48(9)$ & .4492 & .1989 & 294.4 & 0.341 \\
\hline 4.34 & 170 & 11.74 & $1.58(9)$ & .5324 & .1940 & 298.5 & 0.376 \\
\hline 4.75 & 175 & 12.77 & $1.04(9)$ & 6099 & .1829 & 301.0 & 0.409 \\
\hline 5.12 & 180 & 13.76 & $7.16(8)$ & .6790 & .1676 & 302.4 & 0.441 \\
\hline 5.47 & 185 & 14.65 & $5.04(8)$ & .7383 & 1499 & 303.4 & 0.470 \\
\hline 5.80 & 190 & 15.44 & $3.62(8)$ & .7879 & .1315 & 303.9 & 0.496 \\
\hline 6.11 & 195 & 16.10 & $2.64(8)$ & .8286 & .1136 & 304.3 & 0.517 \\
\hline 6.41 & 200 & 16.65 & $1.95(8)$ & .8616 & .0970 & 304.4 & 0.535 \\
\hline 6.71 & 205 & 17.09 & $1.45(8)$ & .8882 & .0821 & 304.5 & 0.549 \\
\hline 7.00 & 210 & 17.44 & $1.09(8)$ & .9096 & .0689 & 304.5 & 0.560 \\
\hline \multicolumn{8}{|c|}{ Solar zenith angle $=60^{\circ}$} \\
\hline-8.51 & 100 & 3.64 & $1.13(15)$ & .0001 & .0001 & 159.6 & 33.9 \\
\hline-7.14 & 105 & 3.66 & $2.84(14)$ & .0003 & .0004 & 160.1 & 56.3 \\
\hline-5.81 & 110 & 3.86 & $7.13(13)$ & .0007 & .0007 & 168.8 & 79.6 \\
\hline-4.54 & 115 & 3.97 & $1.96(14)$ & 0019 & .0020 & 173.2 & 102.0 \\
\hline-3.27 & 120 & 3.94 & $5.60(12)$ & .0050 & .0051 & 170.8 & 114.0 \\
\hline-2.02 & 125 & 4.01 & $1.58(12)$ & 0111 & .0117 & 172.3 & 115.0 \\
\hline-0.80 & 130 & 4.19 & $4.55(11)$ & .0241 & .0277 & 176.6 & 113.0 \\
\hline 0.34 & 135 & 4.65 & $1.36(11)$ & .0535 & .0616 & 188.7 & 110.0 \\
\hline 1.32 & 140 & 5.50 & $4.55(10)$ & .1005 & 1013 & 211.9 & 111.0 \\
\hline 2.14 & 145 & 6.56 & $1.78(10)$ & .1618 & .1351 & 237.9 & 115.0 \\
\hline 2.84 & 150 & 7.61 & $8.21(9)$ & .2356 & .1607 & 257.5 & 120.0 \\
\hline 3.45 & 155 & 8.61 & $4.26(9)$ & .3195 & .1772 & 269.8 & 123.0 \\
\hline 3.99 & 160 & 9.60 & $2.41(9)$ & .4100 & .1848 & 277.0 & 126.0 \\
\hline 4.48 & 165 & 10.62 & $1.46(9)$ & .5017 & .1838 & 281.1 & 128.0 \\
\hline 4.93 & 170 & 11.65 & $9.27(8)$ & .5890 & .1753 & 283.4 & 130.0 \\
\hline 5.33 & 175 & 12.66 & $6.15(8)$ & .6674 & .1613 & 284.7 & 131.0 \\
\hline 5.71 & 180 & 13.60 & $4.21(8)$ & .7345 & 1442 & 285.4 & 132.0 \\
\hline 6.06 & 185 & 14.43 & $2.95(8)$ & .7897 & .1258 & 285.8 & 132.0 \\
\hline 6.40 & 190 & 15.13 & $2.11(8)$ & .8341 & .1078 & 286.1 & 133.0 \\
\hline 6.72 & 195 & 15.69 & $1.53(8)$ & .8692 & .0910 & 286.2 & 133.0 \\
\hline \multicolumn{8}{|c|}{ Solar zenith angle $=90^{\circ}$} \\
\hline-8.50 & 100 & 3.65 & $1.11(15)$ & .0001 & .0002 & 159.9 & 32.3 \\
\hline-7.08 & 105 & 3.40 & $2.89(14)$ & .0003 & .0004 & 149.1 & 54.5 \\
\hline
\end{tabular}


TABLE III-Continued

\begin{tabular}{|c|c|c|c|c|c|c|c|}
\hline$Z$ & $\begin{array}{l}\text { Height } \\
\text { (km) }\end{array}$ & $\begin{array}{c}\text { Scale height } \\
(\mathrm{km})\end{array}$ & $\begin{array}{l}\text { Total number } \\
\text { density } \\
\left(\mathrm{cm}^{-3}\right)\end{array}$ & $\begin{array}{l}\text { Relative number } \\
\text { density } \\
\text { (O) }\end{array}$ & $\begin{array}{l}\text { Relative number } \\
\text { density } \\
\text { (CO) }\end{array}$ & $\begin{array}{c}T \\
\left({ }^{\circ} \mathrm{K}\right)\end{array}$ & $\begin{array}{c}u \text { or } w^{a} \\
\left(\mathrm{~m} \mathrm{sec} \sec ^{-1}\right)\end{array}$ \\
\hline-5.56 & 110 & 3.24 & $6.66(13)$ & .0007 & .0008 & 141.9 & 112.0 \\
\hline-4.04 & 115 & 3.36 & $1.41(13)$ & .0023 & .0025 & 146.2 & 165.0 \\
\hline-2.58 & 120 & 3.46 & $3.19(12)$ & .0071 & .0077 & 149.4 & 182.0 \\
\hline-1.14 & 125 & 3.51 & $7.63(11)$ & .0180 & .0212 & 149.1 & 178.0 \\
\hline 0.26 & 130 & 3.56 & $1.91(11)$ & .0454 & .0515 & 146.1 & 173.0 \\
\hline 1.63 & 135 & 3.86 & $4.76(10)$ & .1061 & .0923 & 148.6 & 181.0 \\
\hline 2.79 & 140 & 4.81 & $1.33(10)$ & .2044 & .1287 & 168.7 & 197.0 \\
\hline 3.71 & 145 & 5.94 & $4.80(9)$ & .3298 & .1516 & 185.5 & 208.0 \\
\hline 4.47 & 150 & 7.09 & $2.13(9)$ & .4649 & .1578 & 195.2 & 215.0 \\
\hline 5.11 & 155 & 8.22 & $1.09(9)$ & .5925 & .1495 & 200.1 & 219.0 \\
\hline 5.67 & 160 & 9.28 & $6.14(8)$ & .7008 & .1317 & 202.4 & 222.0 \\
\hline 6.18 & 165 & 10.19 & $3.68(8)$ & .7851 & .1100 & 203.5 & 223.0 \\
\hline 6.65 & 170 & 10.90 & $2.39(8)$ & .8471 & .0885 & 203.9 & 224.0 \\
\hline \multicolumn{8}{|c|}{ Solar zenith angle $=120^{\circ}$} \\
\hline-8.53 & 100 & 3.74 & $1.12(15)$ & .0002 & .0005 & 163.9 & 18.1 \\
\hline-7.19 & 105 & 3.67 & $3.00(14)$ & .0008 & .0010 & 160.5 & 22.0 \\
\hline-5.80 & 110 & 3.51 & $7.79(13)$ & .0014 & .0016 & 153.1 & 55.6 \\
\hline-4.33 & 115 & 3.33 & $1.91(13)$ & .0040 & .0043 & 144.5 & 86.1 \\
\hline-2.78 & 120 & 3.14 & $4.32(12)$ & .0118 & .0129 & 134.9 & 92.4 \\
\hline-1.14 & 125 & 3.01 & $9.02(11)$ & .0311 & .0306 & 126.1 & 82.6 \\
\hline 0.51 & 130 & 3.09 & $1.76(11)$ & .0725 & .0528 & 123.9 & 74.5 \\
\hline 2.03 & 135 & 3.49 & $3.72(10)$ & .1690 & .0807 & 127.7 & 75.2 \\
\hline 3.34 & 140 & 4.16 & $9.82(9)$ & .3372 & .1031 & 130.8 & 79.4 \\
\hline 4.41 & 145 & 5.10 & $3.32(9)$ & .5445 & .1062 & 131.9 & 82.3 \\
\hline 5.30 & 150 & 6.12 & $1.37(9)$ & .7205 & .0894 & 132.3 & 83.9 \\
\hline 6.05 & 155 & 6.95 & $6.44(8)$ & .8383 & .0661 & 132.4 & 94.7 \\
\hline 6.74 & 160 & 7.49 & $3.24(8)$ & .9075 & .0454 & 132.4 & 85.0 \\
\hline \multicolumn{8}{|c|}{ Solar zenith angle $=180^{\circ}$} \\
\hline-8.56 & 100 & 3.71 & $1.16(15)$ & .0003 & .0030 & 162.4 & -0.023 \\
\hline-7.25 & 105 & 3.86 & $3.02(14)$ & .0024 & .0037 & 168.0 & -0.039 \\
\hline-5.90 & 110 & 3.58 & $8.55(13)$ & .0075 & .0077 & 155.4 & -0.073 \\
\hline-4.42 & 115 & 3.19 & $2.18(13)$ & .0156 & .0135 & 137.4 & -0.078 \\
\hline-2.76 & 120 & 2.91 & $4.65(12)$ & .0286 & .0206 & 123.4 & -0.066 \\
\hline-0.98 & 125 & 2.75 & $8.47(11)$ & .0523 & .0269 & 114.3 & -0.054 \\
\hline 0.83 & 130 & 2.81 & $1.42(11)$ & .1060 & .0353 & 111.2 & -0.049 \\
\hline 2.51 & 135 & 3.19 & $2.63(10)$ & .2475 & .0490 & 112.1 & -0.053 \\
\hline 3.91 & 140 & 3.98 & $6.44(9)$ & .4870 & .0568 & 112.8 & -0.065 \\
\hline 5.01 & 145 & 5.08 & $2.15(9)$ & .7184 & .0494 & 112.9 & -0.083 \\
\hline 5.90 & 150 & 6.00 & $8.83(8)$ & .8612 & .0384 & 113.0 & -0.097 \\
\hline 6.69 & 155 & 6.52 & $4.01(8)$ & .9317 & .0221 & 113.0 & -0.106 \\
\hline
\end{tabular}

${ }^{a}$ Last column gives $u$ for $\theta=60,90,120$, and $w$ for $\theta=0,180$.

man- $\alpha$ data Anderson, 1976; Takacs et al., 1980). Hydrodynamic advection serves to cool the dayside temperatures below previous one-dimensional model values (Part I). Notice also that the temperature gradi- ent across the 140-150-km dayside region and across the terminator is considerably weakened compared to the solar maximum case.

The cooler dayside temperature structure 

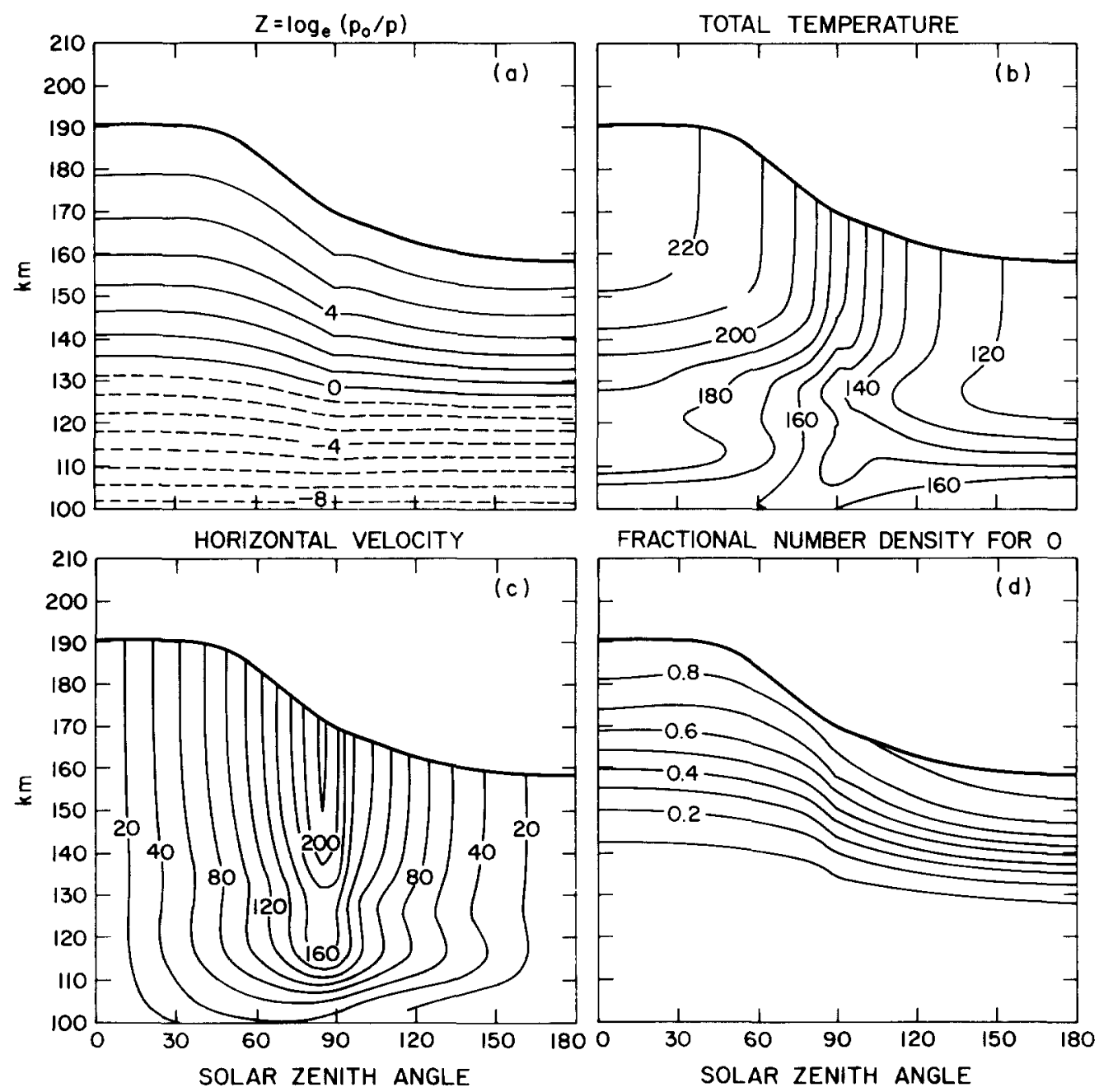

FIG. 12. Solar minimum reference model contour plots. A reduced altitude for the model top boundary $(z=7)$ is shown and is consistent with a collapsed dayside thermosphere. (a) $z=\log _{\mathrm{e}}\left(p_{0} / p\right)$; (b) total temperature in ${ }^{\circ} \mathrm{K}$; (c) horizontal velocity in $\mathrm{m} \mathrm{sec}^{-1}$; (d) fractional number density for $\mathrm{O}$.

results in reduced day-to-night winds, nearly $20-30 \mathrm{~m} \mathrm{sec}^{-1}$ slower at the terminator. These reduced winds have the effect of further isolating the nightside, since less dayside heat is transported and nightside adiabatic heating is diminished. The overall response of the nightside composition and temperatures, however, is quite small; that is, nightside exospheric temperatures are largely unchanged, with a very small reduction in $\mathrm{O}$ densities. EUV heating changes dominate above $140 \mathrm{~km}$, thereby confining the maximum temperature and circulation response to this region. However, the largescale transport of species and heat is largely maintained at lower altitudes (below $z=2$ ) along streamlines for which the wind structure does not greatly change. Our calculations show that Venus's thermospheric structure and circulation are changed relatively little by a factor of 3 reduction in EUV heating over a solar cycle. Thus, $\mathrm{CO}_{2}$ $15-\mu \mathrm{m}$ cooling appears to be an effective buffer against temperature perturbations in 
the Venus thermosphere, giving a solar cycle variation of temperatures much less than that of the Earth (Part I).

We have also examined the time-dependent behavior of the two-dimensional code by turning on solar maximum fluxes instantaneously within the steady-state solar minimum simulation above. From monitoring the dayside temperature profiles for $30-\mathrm{min}$ intervals over the first $3 \mathrm{hr}$ of model integration, and from comparison of all model output fields, we find that in the upper dayside temperatures approach their equilibrium values fastest. The resulting heating subsequently drives terminator horizontal winds that come to equilibrium within the same 3hr period. These winds in turn control the nightside temperature distribution by regulating thermal transport and adiabatic compression. However, time scales greater than one Earth day are required for the nightside temperatures to come to equilibrium.

The redistribution of composition slows down the temperature-wind equilibration. An increase in terminator winds enables nightside densities and temperatures to grow, thereby reducing the day-night pressure gradient which drives these same winds. A balance is obtained as a steady state is approached. Similarly, a reduction in dayside densities weakens the day-night pressure gradient, which causes winds to diminish, thereby providing a means for these same densities and temperatures to increase. This feedback of composition on the dynamics is most simply described as a "virtual temperature effect" (Dickinson and Ridley, 1972), for which dynamic processes tend to eliminate gradients of geopotential height on a constant pressure surface. Hence, motions tend to weaken gradients in $T / \mathrm{m}$, as seen in our Venus calculations.

Equilibrium at low altitudes $(100 \mathrm{~km})$ is approached very slowly (requiring $\gg 1$ Earth day). The "turn on" of solar maximum fluxes does not greatly alter low-altitude winds or temperatures. However, ear- lier drag tests were specifically conducted to observe changes in temperatures and composition as low-altitude winds were permitted to blow uninhibited. Recall that heat was redistributed over all solar zenith angles by these winds, and resulted in the development of nearly constant temperatures at $100 \mathrm{~km}$. Composition was very slowly readjusted and was particularly influential in building up the nightside column. These case runs were integrated for several days before near-equilibrium values could be identified. Feedback among composition, temperature, and wind fields is thus tied to the time scale for hydrodynamics at any given pressure level.

\section{DISCUSSION AND CONCLUSIONS}

The previously developed Venus twodimensional symmetric hydrodynamical model of Dickinson and Ridley (1977) has been revised and improved in light of the Pioneer Venus observations, new data for radiational processes, and new theoretical parameterizations, to give temperature and composition fields that compare quite well with the empirical model atmospheres of Hedin et al. (1983) and Keating et al. (1984). The optimum parameterizations were inferred from a series of sensitivity tests that examined the role played by eddy or wave drag, eddy diffusion, and $15-\mu \mathrm{m}$ radiational cooling in deriving observed day-night contrasts. EUV heating and $15-\mu \mathrm{m}$ cooling parameterizations were adopted from the corrected one-dimensional final reference model calculation of Part I, along with current inputs and fluxes, to give an initial reference calculation neglecting all eddy processes. We then specified eddy drag, diffusion and conduction processes capable of modifying the daynight contrast of composition and temperatures. A weakened circulation was derived from the model sensitivity tests, which provided a great deal of insight concerning the feedback operating among the temperature, wind, and composition fields. Our modeled circulation is constrained solely by the ob- 
served thermospheric structure and remotely measured winds at $115 \mathrm{~km}$.

Wave drag is the primary means by which we are able to obtain day-to-night temperature variations and $\mathrm{O}$ and $\mathrm{CO}$ densities similar to those observed by Pioneer Venus. Eddy viscosity and Rayleigh friction schemes are parameterized within the horizontal momentum equation to prescribe a weakened global-scale circulation. We find that terminator winds are consistent with observed temperature and composition fields about the planet only if maximum horizontal winds are $<230 \mathrm{~m} \mathrm{sec}^{-1}$. Such a reduction of winds by nearly a factor of 2 from those of the Dickinson and Ridley (1977) calculations permits more dissociation products $(\mathrm{O}$ and $\mathrm{CO})$ to remain on the dayside and less to be carried to the nightside, giving $\mathrm{O}, \mathrm{CO}$, and $\mathrm{CO}_{2}$ densities generally within $30-50 \%$ of Pioneer Venus observations. This isolation also serves to enhance the day-night temperature contrast, i.e., dayside temperatures increase and nightside temperatures decrease. Slower winds give less nightside adiabatic compressional heating-the dominant source of nightside heating-which largely balances $15-\mu \mathrm{m}$ cooling to give calculated temperatures above $140 \mathrm{~km}$ closely matching Pioneer Venus values. Changes are smaller at lower levels where the circulation is driven by the IR heating. It was found that drag cannot be used simultaneously with eddy cooling to derive the very cold nightside temperatures observed. Slower winds are sufficient to simulate the cold nightside temperatures; additional eddy diffusion is needed to simulate the observed densities.

A Rayleigh friction scheme is used for the assumed wave drag, presumed to result from breaking internal gravity waves. The vertical profile of this drag coefficient is consistent with the growth of such vertically propagating waves; i.e., the drag profile rate is similar to that used by the $K(z)$ parameterization, and fixes a time scale of $\lambda_{0}=1.2 \times 10^{-4} \mathrm{sec}^{-1}$ at the turbopause.
Such a breaking level corresponds to the average maximum height at which sufficient attenuation occurs due to turbulence to stop further wave growth. Presently, no mechanism besides the weakened circulation given by drag will allow $\mathrm{O}$ and $\mathrm{CO}$ to be retained on the dayside at levels close to observations. The drag regulates the ability of the large-scale winds to transport species to the nightside and to provide an adequate heat source to maintain the observed nightside temperatures. Low-altitude return flow not currently calculated (i.e., below the bottom boundary) could be of help in increasing dayside densities of light species and thus permitting stronger winds and a better nightside distribution of temperatures and integrated heights.

Eddy diffusion is also incorporated in our model calculations to augment the largescale wind effects. Its control over composition (and indirectly temperatures) is secondary to that of the advection effects of the large-scale winds. Eddy diffusion was adjusted to give improved nightside composition, not otherwise obtainable by modifying the momentum drag. It was found that eddy diffusion tended to reduce the observed dayside $\mathrm{O}$ and $\mathrm{CO}$ from that observed, so that an upper limit for the dayside eddy coefficient is inferred $\left(K_{\mathrm{t}}<500\right.$ $\left.\mathrm{m}^{2} \mathrm{sec}^{-1}\right)$. Conversely, nightside densities require $K_{\mathrm{t}}=1000 \mathrm{~m}^{2} \mathrm{sec}^{-1}$ in addition to weakened winds. A factor of 2 enhancement of the eddy coefficient is thus required from day- to nightside. These eddy coefficient values are $<25 \%$ of those used in previous one-dimensional composition models, indicating that the mixing that actually occurs in the Venus two-dimensional model (and presumably the Venus thermosphere) depends on both small-scale turbulent processes and large-scale winds (Shimizu, 1974).

Eddy conduction, consistent with these eddy coefficient profiles, has very little effect upon the calculated two-dimensional model temperatures, and was neglected. As a result, the relatively low (10\%) EUV effi- 
ciency that we find compatible with reasonable eddy cooling $\left(K_{\mathrm{t}}<1000 \mathrm{~m}^{2} \mathrm{sec}^{-1}\right)$ and $15-\mu \mathrm{m}$ cooling $\left(K_{\mathrm{CO}_{2}-\mathrm{O}}<9 \times 10^{-13} \mathrm{~cm}^{3}\right.$ $\mathrm{sec}^{-1}$ ) parameters suggests that either an additional cooling mechanism, or a relaxing of our assumptions about maximum eddy and $15-\mu \mathrm{m}$ cooling, is needed if Fox and Dalgarno (1981) or Hollenbach et al. (1985) model efficiencies (12-18\%) are to be used to calculate observed dayside temperatures.

We realize that Rayleigh friction is not a fundamentally derived parameterization, but rather an ad hoc method to approximate wave-breaking effects on the mean flow. We have also considered an eddy viscosity scheme based on the vertical eddy coefficient used for compositional diffusion. Winds comparable to those obtained from Rayleigh friction cannot be derived with consistent eddy viscosity parameters alone. A final reference model calculation combines optimal Rayleigh friction with eddy viscosity that is consistent with chosen eddy diffusion parameters. This procedure is consistent with our modeling strategy that requires all incorporated eddy processes to be based upon a similar $K(z)$ coefficient and to jointly improve the calculated densities and temperatures.

Any internally consistent wave-drag parameterization like Holton's (1983) must evolve from a much better understanding of the sources of wave activity in the Venus thermosphere. Such a detailed parameterization would depend on specific wave characteristics and local stability parameters and large-scale winds. Further progress in the development of gravity-wave breaking models for Venus's mesospherethermosphere awaits the measurement of wind profiles from 60 to $150 \mathrm{~km}$, as well as specific wave phase speeds and length scales. Schubert and Walterscheid (1984) established that static stability and wind structure are critical parameters for determining the vertical propagation of groundgenerated and cloud-generated gravity waves into the Venus thermosphere. The horizontal winds above $60 \mathrm{~km}$ are too poorly known to warrant a detailed Venus gravity-wave breaking model of the type described by Holton (1983). However, some fraction of the cloud-top super-rotating winds must be present up to $100 \mathrm{~km}$ if lower altitude-generated gravity waves are to propagate into the thermosphere (Schubert and Walterscheid, 1984). Turbulence may also be generated in situ as a result of local wind shear at the terminator (Seiff and Kirk, 1982). However, the largescale flow is stable according to the Richardson number criteria.

Solar minimum calculations were conducted for identical physical parameterizations and inputs (other than reduced solar fiuxes), in order to examine the two-dimensional model behavior over a wide range of solar conditions. The Venus thermosphere is relatively insensitive to solar cycle variations of EUV fluxes. Dayside temperatures $(z>0)$ are reduced by about $70^{\circ} \mathrm{K}$, giving an exospheric temperature of $225^{\circ} \mathrm{K}$ at noon. This value is consistent with that derived from Lyman- $\alpha$ studies from Mariner 5 and 10 data (Anderson, 1976; Takacs et al., 1980). Terminator winds are also reduced by less than $30 \mathrm{~m} \mathrm{sec}^{-1}$, leaving a largely undisturbed nightside thermosphere. In comparison, solar cycle variations result in terrestrial exospheric temperature changes of as much as $900^{\circ} \mathrm{K}$. Evidently, $\mathrm{CO}_{2} 15-\mu \mathrm{m}$ cooling is a very effective buffer against solar perturbations, thereby reducing the atmospheric response from that which would otherwise result from a balance of peak EUV heating and conduction (Part I). As a result, the two-dimensional model circulation changes very little in response to solar flux variations over a solar cycle.

Our simulation of the symmetric (subsolar-to-antisolar) component of the Venus structure and winds is only a partial step in the description of its fully three-dimensional character. Figure 13 illustrates by way of a block diagram the hierarchy of 


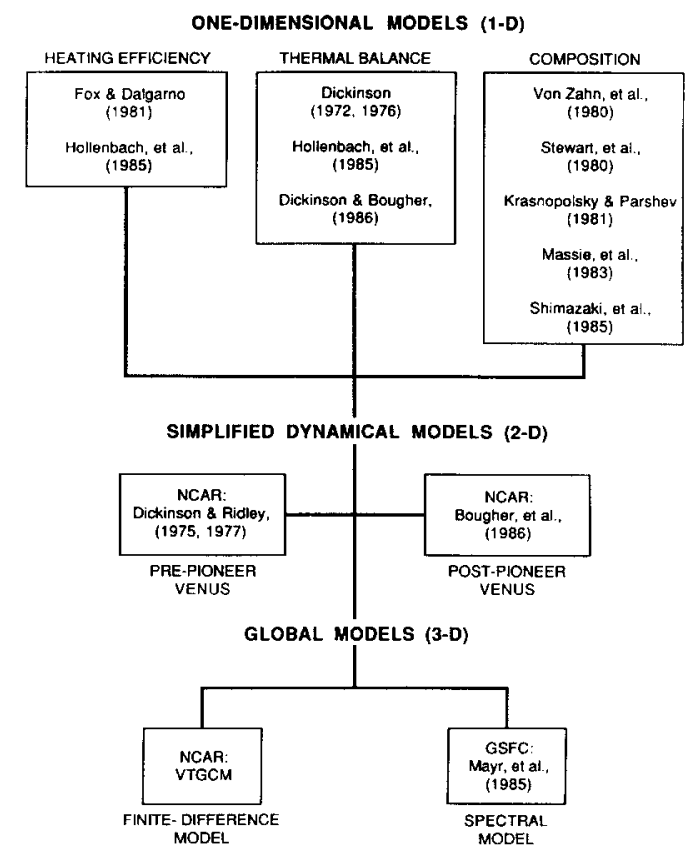

FIG. 13. Block diagram illustrating the hierarchy of previous Venus models which are precursors to the present two-dimensional model study.

previous models which are precursors to the currently discussed Venus model and sensitivity studies. Future work (Part III) will focus on the observed asymmetrical signature of the above fields with a Venus three-dimensional thermospheric general circulation model (VTGCM), described in Bougher et al. (1981) and Bougher (1985). Such a study will build upon the experience gained by the sensitivity studies conducted within the Venus one- and two-dimensional models. The crucial improvement to the VTGCM will be the incorporation of a super-rotating component of the circulation (similar to Mayr et al., 1985) to examine asymmetries in the self-consistently calculated composition and temperature fields, with particular attention given to helium. It is also desirable to incorporate odd-nitrogen species to test the ability of our prescribed circulation to predict the mean behavior of the observed NO night glow (Stewart et al., 1980).

\section{APPENDIX A}

\section{Nonlinear Temperature-Dependent 15- $\mu$ m CoOling Parameterization}

The thermal infrared cooling can now be approximated in the two-dimensional model by the previously calculated Venus day- and nightside reference cooling profiles plus a NLTE cool-to-space formulation for deviations from these references. Deviations depend on departures of the temperature profile and atomic oxygen concentrations from the one-dimensional model values.

In the cool-to-space approximation, the $15-\mu \mathrm{m}$ cooling is proportional to $F(p, T,[\mathrm{O}])$, where for fundamental band temperature dependence,

$$
F(p, T,[\mathrm{O}])=\frac{\exp (-960 / T)}{1+g(p, T, n(\mathrm{O}))} .
$$

The numerator corresponds to the Boltzman factor giving the relative population of the $\nu_{2}$ excited vibrational levels, $n$ is the number concentration of the species in its argument, $p$ is pressure in $\mu$ bar, and $T$ is total temperature. The factor $g$ is adopted from Dickinson and Ridley (1977) with slight modification to incorporate explicitly the $\mathrm{K}_{\mathrm{O}-\mathrm{CO}_{2}}$ and $\mathrm{K}_{\mathrm{CO}_{2}-\mathrm{CO}_{2}}$ rate coefficient expressions:

$$
\begin{aligned}
& g(p, T, n(\mathrm{O})) \cong \frac{0.02}{p} \cdot \exp \left[4 0 . 6 \left(\frac{1}{T_{0}^{1 / 3}}\right.\right. \\
&\left.\left.-\frac{1}{T^{1 / 3}}\right)\right] \times\left(\frac{n\left(\mathrm{CO}_{2}\right)+n(\mathrm{O})}{n\left(\mathrm{CO}_{2}\right)+R \cdot n(\mathrm{O})}\right)
\end{aligned}
$$

where

$$
R=\frac{5.25 \times 10^{-14} \sqrt{T}}{5.20 \times 10^{-15}\left(1+2 \times 10^{-3}(T-220)\right)}
$$

Here, $T_{0}$ denotes the one-dimensional reference model temperature, and $R$ is the ratio of the adopted temperature-dependent expressions for $\mathrm{K}_{\mathrm{O}_{-} \mathrm{CO}_{2}}$ over $\mathrm{K}_{\mathrm{CO}_{2}-\mathrm{CO}_{2}}$ where 


$$
\begin{aligned}
K_{\mathrm{O}-\mathrm{CO}_{2}} & \approx 9 \times 10^{-13} \mathrm{~cm}^{3} \mathrm{sec}^{-1} \text { at } 300^{\circ} \mathrm{K} \\
K_{\mathrm{CO}_{2}-\mathrm{CO}_{2}} & \approx 5.75 \times 10^{-15} \mathrm{~cm}^{3} \mathrm{sec}^{-1} \text { at }
\end{aligned}
$$

We designate $n_{\text {ref }}(\mathrm{O})$ as the reference model $\mathrm{O}$ concentration, and $z=\log _{\mathrm{e}}\left(p_{0} / p\right)$ as $\log$ pressure. The cooling at other temperatures and concentrations is now approximated by

$$
\begin{aligned}
Q_{15}(T, p, n(\mathrm{O}))= & Q_{15}\left(T_{0}, p, n_{\mathrm{ref}}(\mathrm{O})\right) \\
& \times \frac{F(p, T, n(\mathrm{O}))}{F\left(p, T_{0}, n_{\mathrm{ref}}(\mathrm{O})\right)}
\end{aligned}
$$

where $Q_{15}\left(T_{0}, p, n_{\text {ref }}(\mathrm{O})\right)$ is the $15-\mu \mathrm{m}$ cooling for the reference day- or nightside atmosphere. Separate $Q_{15}\left(T_{0}, p, n(\mathrm{O})\right)$ cooling is calculated for dayside and nightside local times based on reference dayside and nightside cooling profiles. Matching is achieved across the terminator by interpolation such that discontinuities are minimized.

The above expression (5) has approximately the correct dependence of the IR cooling on temperature, pressure, and $\mathrm{O}$ concentration and reduces to referencemodel cooling for composition and temperature profiles of either the day- or nightside reference models. The "two-referencetemperature" approach to $15-\mu \mathrm{m}$ cooling helps to parameterize the strong nonlinear dependence of $15-\mu \mathrm{m}$ cooling on temperature for the two-dimensional model. The day-night contrast of Venus temperatures is now more accurately addressed than in Dickinson and Ridley (1977).

\section{ACKNOWLEDGMENTS}

We thank H. Mayr and D. Hunten for useful discussions concerning this research. The comments of C. Fesen, in preparation of this text, are greatly appreciated. Thanks also goes to J. C. Gerard and A. I. F. Stewart for their continued support and interest in this research. S. W. Bougher received partial computing support from NASA Grant NAS2-9130 at the University of Michigan. The authors also thank H. Mayr and another referee for their assistance in evaluating this paper.

\section{REFERENCES}

ANDERSON, D. E., JR. (1976). The Mariner 5 ultraviolet spectrometer experiment: Analysis of hydrogen Lyman- $\alpha$ data. J. Geophys. Res. 81, 1213-1216.
BETZ, A. (1982). Spectroscopic measurements of Mars and Venus with heterodyne techniques. In Vibrational-Rotational Spectroscopy for Planetary Atmospheres, Vol. I, 277-293, NASA Conference Publication 2223.

Bougher, S. W. (1985). Venus Thermospheric Circulation. Ph.D. thesis, University of Michigan, Ann Arbor.

Bougher, S. W., T. E. Cravens, A. F. Nagy, R. G. Roble, AND E. C. Ridley (1981). A finite difference model of the Venus thermospheric circulation. EOS 62, 318.

Cravens, T. E., A. J. Kliore, J. U. Kozyra, and A. F. NAGY (1981). The ionospheric peak on the Venus dayside. J. Geophys. Res. 86, 11,323-11,329.

Dickinson, R. E. (1972). Infrared radiative heating and cooling in the Venusian mesosphere. I. Global mean radiative equilibrium. J. Atmos. Sci. 29, 15311556.

Dickinson, R. E. (1976). Venus mesosphere and thermosphere temperature structure: Global mean radiative and conductive equilibrium. Icarus 27, 479493.

Dickinson, R. E., AND S. W. Bougher (1986). Venus mesosphere and thermosphere: $I$. Heat budget and thermal structure. J. Geophys. Res. 91, 70-80.

Dickinson, R. E., And E. C. Ridley (1972). A numerical solution for the composition of a steady subsolar-to-antisolar circulation with application to Venus. J. Atmos. Sci. 29, 1557-1570.

Dickinson, R. E., ANd E. C. Ridley (1975). A numerical model for the dynamics and composition of the Venusian thermosphere. J. Atmos. Sci. 32, 1219-1231.

Dickinson, R. E., AND E. C. Ridley (1977). Venus mesosphere and thermosphere temperature structure. II. Day-night variations. Icarus 30, 163-178.

Dickinson, R. E., E. C. Ridley, ANd R. G. Roble (1984). Thermospheric general circulation with coupled dynamics and composition. J. Atmos. Sci. 41, 205-219.

Fox, J. L., AND A. Dalgarno (1981). Ionization, luminosity, and heating of the upper atmosphere of Venus. J. Geophys. Res. 86, 629-639.

Gerard, J. C., A. I. F. Stewart, and S. W. Bougher (1981). The altitude distribution of the Venus ultraviolet nightglow and implications on vertical transport. Geophys. Res. Lett. 8, 633-636.

Hedin, A. E., H. B. Niemann, W. T. Kasprazak, AND A. SEIFF (1983). Global empirical model of the Venus thermosphere. J. Geophys. Res. 88, 73-83.

Heroux, L., ANd H. E. Hinteregger (1977). Aeronomical reference spectrum for solar UV below $2000 \AA$. J. Geophys. Res. 82, 3307-3310.

Hollenbach, D. J., S. S. Prasad, and R. C. WhitTEN (1985). The thermal structure of the dayside upper atmosphere of Venus above $125 \mathrm{~km}$. Icarus 64, 205-220. 
Holton, J. R. (1982). The role of gravity wave induced drag and diffusion in the momentum budget of the mesosphere. J. Atmos. Sci. 39, 791-799.

Holton, J. R. (1983). The influence of wave breaking on the general circulation of the middle atmosphere. J. Atmos. Sci. 40, 2497-2507.

Holton, J. R., AND W. M. Wehrbein (1980). A numerical model of the zonal mean circulation of the middle atmosphere. Pure Appl. Geophys. 118, 284 306.

Keating, G. M., A. E. Hedin, J. Y. Nicholson, A. F. Nagy, T. E. Cravens, J. L. Bertaux, U. von ZAHN, AND R. E. DICKINSON (1984). VIRA models of Venus neutral upper atmosphere structure and composition. Presented at Workshop III, Venus Atmosphere and Ionosphere, 25th Plenary Meeting of COSPAR, Graz, Austia, June 22-July 6, 1984.

Krasnopolsky, V. A., and V. A. Parshev (1981). Chemical composition of the atmosphere of Venus. Nature 292, 610-613.

LINDZEN, R. A. (1971). Tides and gravity in the upper atmosphere. In Mesospheric Models and Related Experiments (G. Fiocco, Ed.), Reidel, Hingham, Mass.

LinDZEN, R. S. (1981). Turbulence and stress owing to gravity wave and tidal breakdown. J. Geophys. Res. 86, 9707-9714.

Massie, S. T., D. M. Hunten, and D. T. Sowell (1983). Day and night models of the Venus thermosphere. J. Geophys. Res. 88, 3955-3969.

Matsuno, T. (1983). Quasi one-dimensional model of the middle atmosphere circulation interacting with internal gravity waves. J. Meteorol. Soc. Japan 60, 215-226.

Mayr, H. G., I. Harris, H. B. Niemann, H. C. Brinton, N. W. Spencer, H. A. Taylor, JR., R. E. Hartle, W. R. Hoegy, and D. M. Hunten (1980). Dynamic properties of the thermosphere inferred from Pioneer Venus mass spectrometer measurements. J. Geophys. Res. 85, 7841-7847.

MAYr, H. G., I. HARrIS, AND N. W. SPEnCER (1978). Some properties of the upper atmosphere dynamics. Rev. Geophys. Space Phys. 16, 539-565.

Mayr, H. G., I. Harris, D. R. Stevens-Rayburn, H. B. Niemann, H. A. Taylor, JR., and R. E. HARTLE (1985). On the diurnal variations in the temperature and composition: A three dimensional model with superrotation. In Adv. Space Res. 5, 9, The atmosphere of Venus: Recent findings, Chap. 3, pp. 109-112.

Schoeberl, M. R., ANd D. F. Strobel (1978). The zonally averaged circulation of the middle atmosphere. J. Atmos. Sci. 35, 577-591.

Schubert, G. (1983). General circulation and the dynamical state of the Venus atmosphere. In Venus, Chap. 21, pp. 681-765. Univ. of Arizona Press, Tucson.
SCHUBERT, G., AND R. L. WALTERSChEId (1984). Propagation of small-scale acoustic-gravity waves in the Venus atmosphere. J. Atmos. Sci. 41, 12021213.

SEIFF, A. (1982). Dynamical implications of the observed thermal contrasts in Venus's upper thermosphere. Icarus 51, 574-592.

SEIFF, A., AND D. B. KIRK (1982). Structure of the Venus mesosphere and lower thermosphere from measurements during entry of the Pioneer Venus probes. Icarus 49, 49-70.

SeifF, A., D. B. Kirk, R. E. Young, R. C. Blanchard, J. T. Findley, G. M. Kelly, AND S. C. Sommer (1980). Measurements of the thermal structure and thermal contrasts in the atmosphere of the Venus and related dynamical observations: Results from the four Pioneer Venus probes. J. Geophys. Res. 85, 7903-7933.

Sharma, R. D., AND R. M. Nadile (1981). Carbon Dioxide $\left(\nu_{2}\right)$ Radiance Results Using a New NonEquilibrium Model. AFGL-TR-81-0064.

Shimazaki, T., R. C. Whitten, and H. T. WoodWARD (1984). The dayside Venus ionosphere II. Combined numerical model of ion and neutral composition above $120 \mathrm{~km}$. Icarus 60, 654-674.

ShimizU, M. (1974). Atmospheric mixing in the upper atmospheres of Jupiter and Venus. J. Geophys. Res. 79, 5311-5313.

Simpson, C. J. S. M., P. D. Gait, and J. M. Simmie (1977). The vibrational deactivation of the bending mode of $\mathrm{CO}_{2}$ by $\mathrm{O}_{2}$ and $\mathrm{N}_{2}$. Chem. Phys. Lett. 47, 133-136.

Stewart, A. I. F., J. C. Gerard, D. W. Rusch, and S. W. Bougher (1980). Morphology of the Venus ultraviolet night airglow. J. Geophys. Res. 85, 78617870 .

Takacs, P. Z., A. L. Broadfoot, G. R. SMITH, AND S. Kumar (1980). Mariner 10 observations of hydrogen Lyman- $\alpha$ emission from the Venus exosphere: Evidence of complex structure. Planet. Space Sci. 28, 687-701.

Torr, M. R., D. G. Torr, R. A. Ong, and H. E. Hinteregger (1979). Ionization frequencies for major thermospheric constituents as a function of solar cycle 21. Geophys. Res. Lett. 6, 771-774.

von Zahn, U., K. H. Fricke, D. M. Hunten, D. Krankowsky, K. Mauersberger, and A. O. NiER (1980). The upper atmosphere of Venus during morning conditions. J. Geophys. Res. 85, 78297840.

von Zahn, U., S. Kumar, H. B. NiemanN, and R. PrinN (1983). Composition of the Venus atmosphere. In Venus, Chap. 13, pp. 299-430. Univ. of Arizona Press, Tucson.

Yung, Y. L., ANd W. B. DEMore (1982). Photochemistry of the stratosphere of Venus: Implications for atmospheric evolution. Icarus 51, 199-247. 